quatrième série-tome 43 fascicule 4 juillet-août 2010

$$
\begin{aligned}
& \text { ANNALES } \\
& \text { SCIENTIFIQUES } \\
& \text { de } \\
& \text { L'ECOLE } \\
& \text { NORMALE } \\
& \text { SUPÉRIEURE }
\end{aligned}
$$

Carel FABER \& Sergey SHADRIN \& Dimitri ZVONKINE Tautological relations and the $r$-spin Witten conjecture 
Ann. Scient. Éc. Norm. Sup.

$4^{\mathrm{e}}$ série, t. 43,2010, p. 621 à 658

\title{
TAUTOLOGICAL RELATIONS AND THE $r$-SPIN WITTEN CONJECTURE
}

\section{By Carel FABER, Sergey SHADRIN and Dimitri ZVONKINE}

\begin{abstract}
In [11], A. Givental introduced a group action on the space of Gromov-Witten potentials and proved its transitivity on the semi-simple potentials. In [24, 25], Y.-P. Lee showed, modulo certain results announced by $\mathrm{C}$. Teleman, that this action respects the tautological relations in the cohomology ring of the moduli space $\overline{\mathcal{M}}_{g, n}$ of stable pointed curves.

Here we give a simpler proof of this result. In particular, it implies that in any semi-simple GromovWitten theory where arbitrary correlators can be expressed in genus 0 correlators using only tautological relations, the geometric Gromov-Witten potential coincides with the potential constructed via Givental's group action.

As the most important application we show that our results suffice to deduce the statement of a 1991 Witten conjecture relating the $r$-KdV hierarchy to the intersection theory on the space of $r$-spin structures on stable curves. We use the fact that Givental's construction is, in this case, compatible with Witten's conjecture, as Givental himself showed in [10].
\end{abstract}

RÉSumÉ. - Dans [11], A. Givental a introduit une action de groupe sur l'espace des potentiels de Gromov-Witten et a prouvé sa transitivité sur les potentiels semi-simples. Dans [24, 25], Y.-P. Lee a montré, modulo certains résultats annoncés par $\mathrm{C}$. Teleman, que cette action préserve les relations tautologiques dans l'anneau de cohomologie de l'espace des modules $\bar{M}_{g, n}$ des courbes stables épointées.

Ici nous donnons une démonstration plus simple de ce résultat. Il en découle, entre autres, que si dans une théorie de Gromov-Witten semi-simple on peut exprimer n'importe quel corrélateur en fonction des corrélateurs de genre 0 en utilisant uniquement des relations tautologiques, alors le potentiel de Gromov-Witten géométrique coïncide avec le potentiel construit via l'action du groupe de Givental.

Ces résultats suffisent pour démontrer une conjecture de Witten de 1991 qui relie la hiérarchie $r-\mathrm{KdV}$ à la théorie de l'intersection sur l'espace des structures $r$-spin sur les courbes stables. Nous utilisons pour cela la compatibilité entre la construction de Givental dans ce cas et la conjecture de Witten, compatibilité établie dans [10] par Givental lui-même. 


\section{Introduction}

In Sections 1.1 to 1.4 we give some background information on moduli spaces and tautological relations; the Gromov-Witten potentials, Frobenius manifolds and semi-simplicity; $r$-spin structures and Witten's conjecture; Givental's group action and Y.-P. Lee's universal relations. Finally, in Section 1.5 we formulate our main results.

\subsection{Moduli spaces and tautological relations}

More information on this subject can be found in [13].

1.1.1. Moduli spaces. - For $g, n \in \mathbb{N}, 2-2 g-n<0$, let $\mathcal{M}_{g, n}$ be the moduli space of smooth genus $g$ curves with $n$ distinct marked and numbered points. Let $\bar{M}_{g, n}$ be its DeligneMumford compactification or, in other words, the moduli space of stable genus $g$ curves with $n$ marked and numbered points.

1.1.2. The $\psi$ - and $\kappa$-classes. - There are $n$ naturally defined line bundles $\mathscr{L}_{1}, \ldots, \mathscr{L}_{n}$ over $\bar{M}_{g, n}$. The fiber of $\mathscr{L}_{i}$ over a point $t \in \bar{M}_{g, n}$ is the cotangent line to the $i$-th marked point on the corresponding stable curve $C_{t}$.

Definition 1.1. - The $\psi$-classes $\psi_{1}, \ldots, \psi_{n} \in H^{2}\left(\overline{\mathcal{M}}_{g, n}, \mathbb{Q}\right)$ are the first Chern classes $\psi_{i}=c_{1}\left(\mathscr{L}_{i}\right)$ of the line bundles $\mathscr{L}_{i}$.

Let $\pi: \bar{M}_{g, n+m} \rightarrow \bar{M}_{g, n}$ be the forgetful map; more precisely, $\pi$ forgets the marked points from $n+1$ to $n+m$ and stabilizes the curve by contracting the genus 0 irreducible components with less than three nodes and marked points.

Definition 1.2. - Let $k_{1}, \ldots, k_{m}$ be positive integers, $K=\sum k_{i}$. The $\kappa$-class $\kappa_{k_{1}, \ldots, k_{m}} \in H^{2 K}\left(\bar{M}_{g, n}, \mathbb{Q}\right)$ is defined by

$$
\kappa_{k_{1}, \ldots, k_{m}}=\pi_{*}\left(\psi_{n+1}^{k_{1}+1} \ldots \psi_{n+m}^{k_{m}+1}\right) .
$$

1.1.3. The dual graphs. - Consider a stable curve $C$ of genus $g$ with $n$ marked points. The topological type of $C$ can be described by a graph $G$ obtained by replacing every irreducible component of the curve by a vertex and every node of the curve by an edge. Every marked point is replaced by a tail (an edge that does not lead to any vertex) retaining the same number as the marked point. Each vertex $v$ is labeled by an integer $g_{v}$ : the geometric genus of the corresponding component of $C$. The $g_{v}$ 's and the first Betti number of $G$ add up to $g$.

To a vertex $v$ of $G$ we assign its valency $n_{v}$ : the number of half-edges and tails adjacent to it. The stability of the curve $C$ is equivalent to the stability condition on the graph: $2-2 g_{v}-n_{v}<0$ for every vertex $v$.

In order to avoid problems with automorphisms, we will label all the half-edges of $G$. To $G$ we assign the space

$$
\bar{M}_{G}=\prod_{v} \bar{M}_{g_{v}, n_{v}},
$$

where the product goes over the set of vertices of $G$. The space $\bar{M}_{G}$ comes with a natural map $p: \bar{M}_{G} \rightarrow \bar{M}_{g, n}$ whose image is the closure of the set of stable curves homeomorphic to $C$ (relative to the marked points). Note that $p_{*}\left[\overline{\mathcal{M}}_{G}\right]=|\operatorname{Aut}(G)| \cdot\left[p\left(\overline{\mathcal{M}}_{G}\right)\right]$. 
We can define a cohomology class on $\overline{\mathcal{M}}_{G}$ (and hence on $\bar{M}_{g, n}$ by taking the push-forward of this class under $p$ ) by assigning a class $\kappa_{k_{1}, \ldots, k_{m}}$ to each vertex of $G$ and a power $\psi^{d}$ of the $\psi$-class to each half-edge and each tail of $G$. The class on $\bar{M}_{g, n}$ does not depend on the labeling of the half-edges of $G$.

Definition 1.3. - A stable dual graph or just a dual graph is a graph $G$ with labeled half-edges and numbered tails, satisfying the stability condition, with a set $\left\{k_{1}, \ldots, k_{m}\right\}$ of positive integers assigned to each vertex $v$ and a nonnegative integer $d$ assigned to each half-edge and each tail. The corresponding cohomology class in $H^{*}\left(\overline{\mathscr{M}}_{g, n}, \mathbb{Q}\right)$ is called the tautological class assigned to $G$ and denoted by $[G]$. The genus of a dual graph is the genus of the corresponding stable curves, its degree is the algebraic degree of the corresponding cohomology class, and its dimension is $\operatorname{dim} \bar{M}_{g, n}-$ degree $=3 g-3+n-$ degree.

In the pictures the sets $\left\{k_{1}, \ldots, k_{m}\right\}$ and the integers $d$ will be represented by $\kappa_{k_{1}, \ldots, k_{m}}$ and $\psi^{d}$ respectively, to recall their meanings. The empty sets (corresponding to the cohomology class equal to 1 ) and the integers $d=0$ will be omitted.

1.1.4. Tautological and Gorenstein relations. - Let $G_{g, n}$ be the space of formal rational linear combinations of stable dual graphs of genus $g$ with $n$ tails. Then, extending by linearity the map $G \mapsto[G]$ of Definition 1.3, we obtain a map

$$
\begin{aligned}
\varphi: G_{g, n} & \rightarrow H^{*}\left(\overline{\mathcal{M}}_{g, n}, \mathbb{Q}\right) \\
L & \mapsto[L] .
\end{aligned}
$$

Definition 1.4. - The image of $\varphi$ is called the tautological ring of $\bar{M}_{g, n}$. A linear combination of stable dual graphs is called a tautological relation if it is in the kernel of $\varphi$.

REMARK 1.5. - The tautological ring can also be viewed as a subring of the rational Chow ring. In this paper we only consider the tautological ring as a subring of the cohomology ring. The terms tautological relation and Gorenstein conjecture (see below) are used correspondingly.

EXAmPLE 1.6. - The following linear combination of dual graphs in $G_{1,1}$ is a tautological relation:

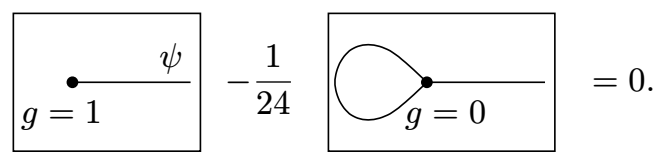

Remark 1.7. - The tautological ring is indeed a ring. Given two linear combinations $L_{1}$ and $L_{2}$ of dual graphs, the class $\left[L_{1}\right] \cup\left[L_{2}\right]$ can be algorithmically expressed as $[L]$ for some linear combination $L$ of dual graphs.

REMARK 1.8. - Although for large $g$ and $n$ the dimension of the tautological ring is much smaller than that of the total cohomology ring of $\bar{M}_{g, n}$, it is quite hard to construct a nontautological cohomology class (see [12]).

ReMAR K 1.9. - The so-called Gorenstein conjecture ([14], Question 5.5, [6], Section 5.1) states that the Poincare duality is a perfect pairing on the tautological ring. 
Definition 1.10. - A linear combination $L \in G_{g, n}$ of dual graphs is called a Gorenstein relation if the intersection of the class $[L]$ with all tautological classes of complementary dimension vanishes.

Thus a tautological relation is always a Gorenstein relation, while the converse is equivalent to the Gorenstein conjecture. Note also that it is possible to check by an algorithm whether $L$ is a Gorenstein relation or not, while (because the Gorenstein conjecture is open) no such algorithm is known for tautological relations.

\subsection{Gromov-Witten potentials and semi-simplicity}

\subsubsection{The Gromov-Witten potential of a point. -}

Definition 1.11. - The genus g Gromov-Witten descendant potential of a point is the formal power series

$$
F_{g}^{\mathrm{pt}}\left(t_{0}, t_{1}, \ldots\right)=\sum_{n \geq 0} \sum_{d_{1}, \ldots, d_{n}}\left\langle\tau_{d_{1}} \ldots \tau_{d_{n}}\right\rangle_{g} \frac{t_{d_{1}} \ldots t_{d_{n}}}{n !}
$$

where

$$
\left\langle\tau_{d_{1}} \ldots \tau_{d_{n}}\right\rangle_{g}=\int_{\overline{\mathcal{M}}_{g, n}} \psi_{1}^{d_{1}} \ldots \psi_{n}^{d_{n}} .
$$

This bracket vanishes unless $\operatorname{dim} \bar{M}_{g, n}=3 g-3+n=\sum d_{i}$. The total Gromov-Witten descendant potential of a point is $F^{\mathrm{pt}}=\sum F_{g}^{\mathrm{pt}} \hbar^{g-1}$ and its exponential $Z^{\mathrm{pt}}=\exp F^{\mathrm{pt}}$ is called the Gromov-Witten partition function of a point.

The partition function $Z^{\mathrm{pt}}$ lies in the space $\mathbb{Q}\left[\left[\hbar^{ \pm 1}, t_{0}, t_{1}, \ldots\right]\right]$. The stability condition implies that for every monomial $M$ of $Z^{\mathrm{pt}}$ we have $2 \operatorname{deg}_{\hbar} M+\operatorname{deg}_{t} M>0$.

1.2.2. An axiomatization of genus 0 Gromov-Witten potentials. - A formal genus 0 Gromov-Witten potential is defined to model certain properties of $F_{0}^{\mathrm{pt}}$ and those of genus 0 Gromov-Witten potentials of more general target Kähler manifolds $X$ (see, for instance, [11], [9] or [8]). We restrict our considerations to the even part of the cohomology of $X$. In our description we explain in brackets the geometric aspects that motivate the axiomatic definitions.

Let $V$ be a complex vector space [the space $H^{\text {even }}(X, \mathbb{C})$ ] in which we choose for convenience a basis $A$. The space $V$ is endowed with a distinguished element 1 [the cohomology class 1], which, unless otherwise stated, is chosen to be the first vector of the basis. The space $V$ is also endowed with a nondegenerate symmetric bilinear form $\eta$ [the Poincaré pairing]. The coefficients of $\eta$ in the basis will be denoted by $\eta_{\mu \nu}$ and the coefficients of the inverse matrix by $\eta^{\mu \nu}$. Given a triple $(V, 1, \eta)$ we can define a genus 0 Gromov-Witten potential.

Let $M$ be a neighborhood of the origin in $V$. Let $F_{0}$ be a power series in variables $t_{d}^{\mu}$, $d=1,2,3, \ldots, \mu \in A$, whose coefficients are analytic functions on $M$ in variables $t_{0}^{\mu}$. The coefficients of $F_{0}$ are denoted by

$$
F_{0}=\sum_{n \geq 0} \sum_{\substack{d_{1}, \ldots, d_{n} \geq 0 \\ \mu_{1}, \ldots, \mu_{n} \in A}}\left\langle\tau_{d_{1}, \mu_{1}} \ldots \tau_{d_{n}, \mu_{n}}\right\rangle \frac{t_{d_{1}}^{\mu_{1}} \ldots t_{d_{n}}^{\mu_{n}}}{n !} .
$$

$4^{\text {e }}$ SÉRIE - TOME $43-2010-\mathrm{N}^{\mathrm{o}} 4$ 
Definition 1.12. - A power series $F_{0}$ in the variables $t_{d}^{\mu}$ is called a formal genus 0 Gromov-Witten potential if it satisfies the string equation:

$$
\frac{\partial F_{0}}{\partial t_{0}^{1}}=\frac{1}{2} \sum_{\mu, \nu \in A} \eta_{\mu, \nu} t_{0}^{\mu} t_{0}^{\nu}+\sum_{\substack{d \geq 1 \\ \mu \in A}} t_{d+1}^{\mu} \frac{\partial F_{0}}{\partial t_{d}^{\mu}}
$$

the dilaton equation:

$$
\frac{\partial F_{0}}{\partial t_{1}^{1}}=\sum_{\substack{d \geq 1 \\ \mu \in A}} t_{d}^{\mu} \frac{\partial F_{0}}{\partial t_{d}^{\mu}}-2 F_{0}
$$

and the topological recursion relation:

$$
\forall a, b, c \in \mathbb{N}, \mu, \nu, \rho \in A, \quad \frac{\partial^{3} F_{0}}{\partial t_{a+1}^{\mu} \partial t_{b}^{\nu} \partial t_{c}^{\rho}}=\sum_{\lambda, \sigma \in A} \frac{\partial^{2} F_{0}}{\partial t_{a}^{\mu} \partial t_{0}^{\lambda}} \eta^{\lambda, \sigma} \frac{\partial^{3} F_{0}}{\partial t_{0}^{\sigma} \partial t_{b}^{\nu} \partial t_{c}^{\rho}} .
$$

The open set $M$ is called a Frobenius manifold ${ }^{(1)}$.

REMARK 1.13. - The reason for requiring $F_{0}$ to be analytic in the variables $t_{0}^{\mu}$ will become clear when we define semi-simple Gromov-Witten potentials.

Remark 1.14. - If $X$ is a Kähler manifold and $E \subset H_{2}(X, \mathbb{Z})$ is its semi-group of effective 2 -cycles, one usually considers Gromov-Witten potentials with coefficients not in $\mathbb{C}$, but in the Novikov ring of power series of the form

$$
\sum_{D \in E} c_{D} Q^{D}, \quad c_{D} \in \mathbb{C} .
$$

We will mostly work with Gromov-Witten potentials over $\mathbb{C}$, since we do not need Novikov rings for our main application, namely the Witten conjecture. However, we indicate in remarks the modifications that must be made when working over a Novikov ring. A detailed introduction to formal Gromov-Witten potentials, including a discussion of Novikov rings, can be found in [27].

EXAmple 1.15. - Let $V$ be a 1-dimensional vector space with coordinate $t_{0}$ endowed with the quadratic form $\eta=\left(d t_{0}\right)^{2}$ and a unit element $1=\frac{1}{\alpha} \frac{\partial}{\partial t_{0}}$. Then the genus 0 part of the rescaled Gromov-Witten potential of the point

$$
F^{\mathrm{pt}, \alpha}=\sum \hbar^{g-1} \alpha^{\sum d_{i}+2 g-2}\left\langle\tau_{d_{1}} \ldots \tau_{d_{n}}\right\rangle \frac{t_{d_{1}} \ldots t_{d_{n}}}{n !}
$$

is a formal Gromov-Witten genus 0 potential for this space $V$.

Let $V$ be a $k$-dimensional vector space with coordinates $t_{0}^{1}, \ldots, t_{0}^{k}$, endowed with the quadratic form $\eta=\sum\left(d t_{0}^{\mu}\right)^{2}$ and a unit element $1=\sum_{\mu=1}^{k} \frac{1}{\alpha_{\mu}} \frac{\partial}{\partial t_{0}^{\mu}}$. The genus 0 part of the sum

$$
F^{\alpha_{1}, \ldots, \alpha_{k}}=\sum_{\mu=1}^{k} F^{\mathrm{pt}, \alpha_{\mu}}\left(t_{d}^{\mu}\right)
$$

is a formal Gromov-Witten genus 0 potential for this space $V$.

(1) Sometimes the definition of a Frobenius manifold also includes an Euler field and $F_{0}$ is required to satisfy certain homogeneity conditions with respect to this field. In other sources Frobenius manifolds with an Euler field are called conformal. 
In terminology coming from physics, the Taylor coefficients $\left\langle\tau_{d_{1}, \mu_{1}} \ldots \tau_{d_{n}, \mu_{n}}\right\rangle_{g}$ of Gromov-Witten potentials are called correlators, while the elements of the basis $A$ are called primary fields.

\subsubsection{Semi-simplicity}

Definition 1.16. - A Frobenius algebra is a commutative associative algebra $V$ endowed with a nondegenerate bilinear form $($,$) satisfying (a b, c)=(a, b c)$. It is semi-simple if $V$ is a direct sum of one-dimensional algebras.

The idempotents $v_{1}, \ldots, v_{k}$ of a semi-simple Frobenius algebra are automatically pairwise orthogonal with respect to the bilinear form (, ). Their square lengths $\alpha_{\mu}=\left(v_{\mu}, v_{\mu}\right)$ are invariants of a semi-simple Frobenius algebra.

Given a formal genus 0 Gromov-Witten potential $F_{0}\left(t_{d}^{\mu}\right)$, let $f_{0}\left(t_{0}^{\mu}\right)$ be the series obtained from $F_{0}$ by setting $t_{d}^{\mu}=0$ for $d \geq 1$. On every tangent space to the Frobenius manifold $M$, one defines an algebra via the structural constants

$$
C_{\mu_{1}, \mu_{2}}^{\mu_{3}}\left(t_{0}^{\mu}\right)=\sum_{\nu} \frac{\partial^{3} f_{0}}{\partial t_{0}^{\mu_{1}} \partial t_{0}^{\mu_{2}} \partial t_{0}^{\nu}} \eta^{\nu, \mu_{3}}
$$

depending on $t_{0}^{\mu} \in M$. Together with the bilinear form $\eta$, one gets the structure of a Frobenius algebra in every tangent space to $M$.

Definition 1.17. - A genus 0 Gromov-Witten potential $F_{0}$ is called semi-simple if the above Frobenius algebra structure is semi-simple for generic $t_{0}^{\mu} \in M$. The rank of $F_{0}$ is the dimension of $V$.

Remark 1.18. - A genus 0 Gromov-Witten potential defined over a Novikov ring $R$ is called semi-simple if the algebra structure at a generic point is semi-simple over the algebraic closure of the field of fractions of $R$.

Example 1.19. - Let $F$ be the Gromov-Witten potential of $\mathbb{C P}^{1}$. Set $x=t_{0}^{1}, y=t_{0}^{\omega}$, where $1 \in H^{0}\left(\mathbb{C P}^{1}\right)$ and $\omega \in H^{2}\left(\mathbb{C P}^{1}\right)$ form the natural basis of $H^{*}\left(\mathbb{C P}^{1}\right)$. Then

$$
f_{0}(x, y)=\frac{x^{2} y}{2}+Q e^{y}
$$

The Frobenius algebras are semi-simple at all points in the neighborhood of the origin, the (non-normalized) idempotents at the origin being

$$
\frac{\partial}{\partial x} \pm Q^{-1 / 2} \frac{\partial}{\partial y}
$$

Let $F$ be the Gromov-Witten potential of $\mathbb{C P}^{2}$. Set $x=t_{0}^{1}, y=t_{0}^{\omega}, z=t_{0}^{\omega^{2}}$, where $1 \in H^{0}\left(\mathbb{C P}^{1}\right), \omega \in H^{2}\left(\mathbb{C P}^{1}\right), \omega^{2} \in H^{4}\left(\mathbb{C P}^{2}\right)$ form the natural basis of $H^{*}\left(\mathbb{C P}^{2}\right)$. Then

$$
f_{0}(x, y, z)=\frac{x^{2} z}{2}+\frac{x y^{2}}{2}+\sum_{d \geq 1} Q^{d} N_{d} \frac{z^{3 d-1}}{(3 d-1) !} e^{d y},
$$

$4^{\mathrm{e}}$ SÉRIE - TOME $43-2010-\mathrm{N}^{\circ} 4$ 
where $N_{d}$ is the number of rational degree $d$ curves through $3 d-1$ generic points on $\mathbb{C P}^{2}$. The Frobenius algebras are semi-simple at all points in the neighborhood of the origin, the (non-normalized) idempotents at the origin being

$$
\frac{\partial}{\partial x}+Q^{-1 / 3} \frac{\partial}{\partial y}+Q^{-2 / 3} \frac{\partial}{\partial z}
$$

with three possible choices of the cubic root of $Q$.

Let $F$ be the simultaneous solution of the string equation and of the 3-KdV hierarchy. This is the Gromov-Witten potential that appears in Witten's conjecture for $r=3$, see Section 1.3.3. Set $x=t_{0}^{0}, y=t_{0}^{1}$. Then

$$
f_{0}(x, y)=\frac{x^{2} y}{2}+\frac{y^{4}}{72}
$$

The Frobenius algebras are semi-simple outside the discriminant $\{y=0\}$, the (nonnormalized) idempotents being

$$
\frac{\partial}{\partial x} \pm \sqrt{\frac{3}{y}} \frac{\partial}{\partial y}
$$

\subsection{Witten's $r$-spin conjecture}

\subsubsection{The space of $r$-spin structures. -}

Definition 1.20. - An $r$-spin structure on a smooth curve $C$ with $n \geq 1$ marked points $x_{1}, \ldots, x_{n}$ is a line bundle $\mathcal{T}$ together with an identification

$$
\mathcal{T}^{\otimes r} \simeq K\left(-\sum a_{i} x_{i}\right),
$$

where $K$ is the cotangent line bundle and the integers $a_{i} \in\{0, \ldots, r-1\}$ are chosen in such a way that $2 g-2-\sum a_{i}$ is divisible by $r$.

Two $r$-spin structures $\left(C_{1}, \mathcal{J}_{1}\right)$ and $\left(C_{2}, \mathcal{T}_{2}\right)$ are isomorphic if there exists an isomorphism between $C_{1}$ and $C_{2}$ that identifies the marked points and lifts to an isomorphism of line bundles $\mathcal{J}_{1}$ and $\mathcal{T}_{2}$ commuting with the identification of their $r$-th powers with $K\left(-\sum a_{i} x_{i}\right)$.

The moduli space of $r$-spin structures on smooth curves up to isomorphisms has a natural compactification $\overline{\mathcal{M}}_{g ; a_{1}, \ldots, a_{n}}^{1 / r}$ with a forgetful map $p: \overline{\mathcal{M}}_{g ; a_{1}, \ldots, a_{n}}^{1 / r} \rightarrow \overline{\mathcal{M}}_{g, n}$ (see $\left.[1,4,16]\right)$.

1.3.2. Witten's class. - Using the line bundle $\mathcal{T}$ and the forgetful map $p$, Polishchuk and Vaintrob [31] constructed a cohomology class $c_{W}\left(a_{1}, \ldots, a_{n}\right)$ of pure dimension on $\bar{M}_{g, n}$, now called Witten's class (later Chiodo [3] proposed a simpler construction).

Assuming that $H^{0}(C, \mathcal{T})=0$ for every stable curve $C$, we find that $V=H^{1}(C, \mathcal{J})$ is a vector bundle over $\overline{\mathcal{M}}_{g ; a_{1}, \ldots, a_{n}}^{1 / r}$. Then Witten's class is defined by

$$
c_{W}\left(a_{1}, \ldots, a_{n}\right)=\frac{1}{r^{g}} p_{*}\left(c_{\text {top }}\left(V^{\vee}\right)\right) .
$$

In other words: take the dual vector bundle of $V$, take its Euler (or top Chern) class, take its pushforward to $\bar{M}_{g, n}$, and divide by $r^{g}$. In the general case, when $H^{0}(C, \mathcal{T})$ does not vanish identically, Witten's class has a much more intricate definition that we do not recall here.

Witten's class satisfies the following properties, proved in [30]:

1 . If $a_{i}=r-1$ for some $i$, then $c_{W}\left(a_{1}, \ldots, a_{n}\right)=0$. 
2. Let $p: \bar{M}_{g, n+1} \rightarrow \bar{M}_{g, n}$ be the forgetful map. Then

$$
p^{*}\left(c_{W}\left(a_{1}, \ldots, a_{n}\right)\right)=c_{W}\left(a_{1}, \ldots, a_{n}, 0\right) .
$$

3. Let $r: \bar{M}_{g-1, n+2} \rightarrow \overline{\mathcal{M}}_{g, n}$ and $q: \overline{\mathcal{M}}_{g_{1}, n_{1}+1} \times \overline{\mathcal{M}}_{g_{2}, n_{2}+1} \rightarrow \overline{\mathcal{M}}_{g, n}$ be the gluing mappings to the boundary components of $\overline{\mathcal{M}}_{g, n}$. In the second case we assume, for simplicity, that the $n_{1}$ marked points on the component of genus $g_{1}$ have numbers $1, \ldots, n_{1}$, while the $n_{2}$ marked points on the component of genus $g_{2}$ have numbers $n_{1}+1, \ldots, n$. Then we have

$$
q^{*}\left(c_{W}\left(a_{1}, \ldots, a_{n}\right)\right)=\sum_{a^{\prime}+a^{\prime \prime}=r-2} c_{W}\left(a_{1}, \ldots, a_{n_{1}}, a^{\prime}\right) \times c_{W}\left(a^{\prime \prime}, a_{n_{1}+1}, \ldots, a_{n}\right),
$$

(where at most one term of the sum is actually nonzero, because of the condition $2 g_{1}-2-\sum_{i=1}^{n_{1}} a_{i}-a^{\prime} \equiv 2 g_{2}-2-\sum_{i=n_{1}+1}^{n} a_{i}-a^{\prime \prime} \equiv 0 \bmod r$ ), and

$$
r^{*}\left(c_{W}\left(a_{1}, \ldots, a_{n}\right)\right)=\sum_{a^{\prime}+a^{\prime \prime}=r-2} c_{W}\left(a_{1}, \ldots, a_{n}, a^{\prime}, a^{\prime \prime}\right)
$$

REMARK 1.21. - These properties actually say that $c_{W}$ satisfies the axioms of a cohomological field theory [22] with an $(r-1)$-dimensional space of primary fields.

1.3.3. The r-spin Gromov-Witten potential. - The generating series studied in Witten's conjecture is

$$
F^{[r]}=\sum_{\substack{g \geq 0 \\ n \geq 1}} \hbar^{g-1} \sum_{\substack{d_{1}, \ldots, d_{n} \\ a_{1}, \ldots, a_{n}}} \int_{\bar{M}_{g, n}} c_{W}\left(a_{1}, \ldots, a_{n}\right) \psi_{1}^{d_{1}} \ldots \psi_{n}^{d_{n}} \cdot \frac{t_{d_{1}}^{a_{1}} \ldots t_{d_{n}}^{a_{n}}}{n !}
$$

Motivated by the properties of Witten's class, we introduce the basis $A=\{0, \ldots, r-2\}$ with 0 its distinguished element, the vector space $V$ spanned by $A$, and the quadratic form $\eta^{a b}=\delta^{a+b, r-2}$.

(There is an unfortunate clash of notation with the Givental theory, where the distinguished primary field is usually denoted by 1 , a convention that we follow in our paper except in the applications to Witten's conjecture.)

Theorem (see [19]). - The genus 0 part of $F^{[r]}$ is a formal genus 0 Gromov-Witten potential. Moreover, it is semi-simple.

WitTEn's CONJECTURE ([35]). - It states that the series $F^{[r]}$ is a solution of the $r$-KdV (or $r$-th higher Gelfand-Dikii) hierarchy.

Useful references on Witten's $r$-spin conjecture also include [1, 2, 3, 4, 17, 18, 20, 21, 33].

$4^{\mathrm{e}}$ SÉRIE - TOME $43-2010-\mathrm{N}^{\mathrm{o}} 4$ 


\subsection{Givental's group action}

1.4.1. The operators. - As before, let $V$ be a vector space with a nondegenerate quadratic form $\eta$, a distinguished element 1 and a basis $A$.

Let $r(z)=\sum_{l \geq 1} r_{l} z^{l}$ and $s\left(z^{-1}\right)=\sum_{l \geq 1} s_{l} z^{-l}$ be power series in $z$ and $z^{-1}$ respectively, whose coefficients $r_{l}$ and $s_{l}$ are endomorphisms of $V$. We require the endomorphisms $r_{l}$ and $s_{l}$ to be self-adjoint for $l$ odd and skew-self-adjoint for $l$ even with respect to $\eta$.

The matrices of $s_{l}$ and $r_{l}$ in the basis $A$ will be denoted by $\left(s_{l}\right)_{\mu}^{\nu}$ and $\left(r_{l}\right)_{\mu}^{\nu}$. We will also need the matrices

$$
\begin{aligned}
\left(s_{l}\right)_{\mu \nu} & =\sum_{\rho} \eta_{\mu \rho}\left(s_{l}\right)_{\nu}^{\rho}, & \left(s_{l}\right)^{\mu \nu} & =\sum_{\rho} \eta^{\mu \rho}\left(s_{l}\right)_{\rho}^{\nu}, \\
\left(r_{l}\right)_{\mu \nu} & =\sum_{\rho} \eta_{\mu \rho}\left(r_{l}\right)_{\nu}^{\rho}, & \left(r_{l}\right)^{\mu \nu} & =\sum_{\rho} \eta^{\mu \rho}\left(r_{l}\right)_{\rho}^{\nu} .
\end{aligned}
$$

The matrices $\left(s_{l}\right)_{\mu \nu},\left(r_{l}\right)_{\mu \nu},\left(s_{l}\right)^{\mu \nu}$, and $\left(r_{l}\right)^{\mu \nu}$ are symmetric for odd $l$ and skew-symmetric for even $l$.

Definition 1.22. - The quantizations $\widehat{r}$ and $\widehat{s}$ of $r$ and $s$ are the differential operators

$$
\begin{aligned}
\widehat{r}= & -\sum_{\substack{l \geq 1 \\
\mu}}\left(r_{l}\right)_{1}^{\mu} \frac{\partial}{\partial t_{l+1}^{\mu}}+\sum_{\substack{d \geq 0, l \geq 1 \\
\mu, \nu}}\left(r_{l}\right)_{\nu}^{\mu} t_{d}^{\nu} \frac{\partial}{\partial t_{d+l}^{\mu}}+\frac{\hbar}{2} \sum_{\substack{d_{1}, d_{2} \geq 0 \\
\mu_{1}, \mu_{2}}}(-1)^{d_{1}+1}\left(r_{d_{1}+d_{2}+1}\right)^{\mu_{1} \mu_{2}} \frac{\partial^{2}}{\partial t_{d_{1}}^{\mu_{1}} \partial t_{d_{2}}^{\mu_{2}}} . \\
\widehat{s}= & -\sum_{\mu}\left(s_{1}\right)_{1}^{\mu} \frac{\partial}{\partial t_{0}^{\mu}}+\frac{1}{\hbar} \sum_{d, \mu}\left(s_{d+2}\right)_{1, \mu} t_{d}^{\mu}-\frac{1}{2 \hbar}\left(s_{3}\right)_{1,1} \\
& +\sum_{\substack{d, l \\
\mu, \nu}}\left(s_{l}\right)_{\nu}^{\mu} t_{d+l}^{\nu} \frac{\partial}{\partial t_{d}^{\mu}}+\frac{1}{2 \hbar} \sum_{\substack{d_{1}, d_{2} \\
\mu_{2}, \mu_{2}}}(-1)^{d_{1}}\left(s_{d_{1}+d_{2}+1}\right)_{\mu_{1}, \mu_{2}} t_{d_{1}}^{\mu_{1}} t_{d_{2}}^{\mu_{2}} .
\end{aligned}
$$

In our paper these complicated operators appear out of the blue. We refer to Givental's work [9], for a strikingly concise way to obtain them from a formalism of quantization of quadratic Hamiltonians.

In addition to these operators, to any $\Psi \in \mathrm{GL}(V)$ we assign the differential operator

$$
\widehat{\Psi}=\sum_{d, \mu, \nu} \Psi_{\nu}^{\mu} t_{d}^{\nu} \frac{\partial}{\partial t_{d}^{\mu}} .
$$

This operator simply performs the linear change of variables given by $\Psi$ in every group of variables $t_{d}^{1}, \ldots, t_{d}^{k}$.

1.4.2. Genus expansion of semi-simple potentials. - Let $F_{0}$ be a semi-simple formal genus 0 Gromov-Witten potential and $M$ the corresponding Frobenius manifold.

Assume that the scalar squares of the idempotents of the Frobenius algebra at some point of $M$ equal $\alpha_{1}, \ldots, \alpha_{k}$. Let $Z^{\alpha_{1}, \ldots, \alpha_{k}}=\exp \left(F^{\alpha_{1}, \ldots, \alpha_{k}}\right)$, see Equation (2).

Theorem (see [11] Def. 6.8 and [9] Thm 1 and corollary). - There exist power series $r(z)$ and $s\left(z^{-1}\right)$ and an endomorphism $\Psi$ of $V$ such that $F_{0}$ is the genus 0 part of

$$
\ln \left(e^{-\widehat{s}} \widehat{\Psi} e^{\widehat{r}} Z^{\alpha_{1}, \ldots, \alpha_{k}}\right) .
$$


In other words, every semi-simple formal genus 0 potential can be obtained from the "standard" potential $F^{\alpha_{1}, \ldots, \alpha_{k}}$ via the action of Givental's operators.

The notion of higher genus Gromov-Witten potentials is not axiomatized in full generality. However there is a way to define formal Gromov-Witten potentials whose genus 0 part is semi-simple using Givental's operators.

Definition 1.23. - Let $F_{0}$ be a semi-simple formal genus 0 potential. Choose $r, s$ and $\Psi$ as in the theorem above. Then

$$
F=\ln \left(e^{-\widehat{s}} \widehat{\Psi} e^{\widehat{r}} Z^{\alpha_{1}, \ldots, \alpha_{k}}\right)
$$

is called a formal Gromov-Witten potential or a genus expansion of $F_{0}$.

Remark 1.24. - Let $\breve{M}$ be the open subset of the Frobenius manifold $M$ where the tangent Frobenius algebras are semi-simple. Let hol $\left(t_{0}^{\mu}\right)$ be the space of holomorphic functions in variables $t_{0}^{\mu}$ defined on $\breve{M}$. Then, as we explain in more detail in Section 4, a formal Gromov-Witten potential lies in the space $\hbar^{-1} \operatorname{hol}\left(t_{0}^{\mu}\right) \mathbb{C}\left[\left[\hbar, t_{1}^{\mu}, t_{2}^{\mu}, \ldots\right]\right]$. In particular, although $F_{0}$ can, by definition, be decomposed into a power series in variables $t_{0}^{\mu}, t_{1}^{\mu}, \ldots$ at the origin of $V$, this is not necessarily the case for its genus expansion.

REMARK 1.25. - The series $s$ and the endomorphism $\Psi$ in Givental's theorem are uniquely determined by $F_{0}$. On the other hand, the series $r$ is not determined in a unique way; as a consequence, the genus expansion is not unique either. We are now going to introduce the homogeneity condition that lifts the ambiguity.

Let $F=\sum \hbar^{g-1} F_{g}$ be the Gromov-Witten potential of a target Kähler manifold $X$. Choose a homogeneous basis $A$ of $H^{\text {even }}(X)$. To each variable $t_{d}^{\mu}$ we assign its weight $w\left(t_{d}^{\mu}\right)=d+\operatorname{deg}(\mu)-1$, where $\operatorname{deg}(\mu)$ is the algebraic degree of $\mu \in A$. Further, introduce a weight function on the Novikov ring: $w\left(Q^{D}\right)=-\left\langle D, c_{1}(T X)\right\rangle$ for an effective divisor $D$. Finally, denote by $\operatorname{dim} X$ the dimension of $X$.

The expected dimension of $X_{g, n, D}$ is $n+(1-g)(\operatorname{dim} X-3)+\left\langle D, c_{1}(T X)\right\rangle$. Therefore the correlator $\left\langle\tau_{d_{1}, \mu_{1}} \ldots \tau_{d_{n}, \mu_{n}}\right\rangle_{g, D}$ vanishes unless

$$
\begin{aligned}
\sum d_{i}+\sum \operatorname{deg}\left(\mu_{i}\right) & =n+(1-g)(\operatorname{dim} X-3)+\left\langle D, c_{1}(T X)\right\rangle \\
\Leftrightarrow \sum w\left(t_{d_{i}}^{\mu_{i}}\right)+w\left(Q^{D}\right) & =(1-g)(\operatorname{dim} X-3) .
\end{aligned}
$$

In other words, $F_{g}$ is quasihomogeneous of total weight $(1-g)(\operatorname{dim} X-3)$.

This property is formalized in the following definition.

Definition 1.26. - Introduce a weight map $w: A \rightarrow \mathbb{Q}$ such that $w(1)=-1$, let $w\left(t_{d}^{\mu}\right)=d+w(\mu)$. When working over a Novikov ring, also introduce a weight valuation function on it. Let $\operatorname{dim} \in \mathbb{Q}$ be a constant.

A formal Gromov-Witten potential $F=\sum \hbar^{g-1} F_{g}$ is called homogeneous with respect to $w$ and dim if every $F_{g}$ is quasihomogeneous of total weight $(1-g)(\operatorname{dim}-3)$. 
REMARK 1.27. - In the framework of Frobenius manifolds it is more natural not to attribute weights to the variables $Q$, since they are part of the ground ring. Instead, denote by $c^{\mu}$ the coordinates of the class $c_{1}(T X)$ in basis $A$. Of course, $c^{\mu}$ can be different from 0 only if $\mu$ is of degree 1 , so that the weight of $t_{0}^{\mu}$ equals 0 . We then attribute a weight $c^{\mu}$ to the exponential $\exp \left(t_{0}^{\mu}\right)$. It follows from the divisor axiom that the Gromov-Witten potential is still homogeneous with respect to these new weights, but only up to terms corresponding to degree 0 maps.

Theorem (Givental). - A homogeneous formal genus 0 Gromov-Witten potential has a unique homogeneous genus expansion.

Proposition 6.7.c in [11] proves the unicity of the series $r$, while the fact that the series $s$ is uniquely determined by $F_{0}$ follows from the results of Section 5 in [11] (see also Thm. 2 in [9]).

Givental conjectured that if $F_{0}$ is the geometric genus 0 potential of a target space $X$, then the total geometric Gromov-Witten potential of $X$ coincides with the homogeneous genus expansion of its genus 0 part.

1.4.3. Universal relations. - Let $F=\sum \hbar^{g-1} F_{g}$ be the geometric Gromov-Witten potential of some target Kähler manifold $X, A$ a basis of $H^{*}(X)$ and $\eta$ the Poincaré pairing. Let $L \in G_{g, n}$ be a linear combination of dual graphs. Let $X_{g, n+n^{\prime}, D}=\overline{\mathcal{M}}_{g, n+n^{\prime}}(X, D)$, where $D \in E$ is an effective 2-cycle. Denote by $\left[X_{g, n+n^{\prime}, D}\right]$ the virtual fundamental class, and let $p: X_{g, n+n^{\prime}, D} \rightarrow \bar{M}_{g, n}$ be the forgetful map.

Proposition 1.28. - The simultaneous vanishing of the integrals

$$
\sum_{D \in E} Q^{D} \int_{\left[X_{g, n+n^{\prime}, D}\right]} p^{*}([L]) \prod_{i=1}^{n+n^{\prime}} \psi_{i}^{d_{i}} \mathrm{ev}_{i}^{*}\left(\mu_{i}\right)
$$

for all $n^{\prime}, D$ and for all $d_{1}, \ldots, d_{n+n^{\prime}}$ and $\mu_{1}, \ldots, \mu_{n+n^{\prime}}$ is equivalent to a countable family of partial differential equations with constant coefficients on $F_{0}, \ldots, F_{g}$.

This proposition is not new. We recall the construction of the family of PDEs in Section 2.1.3, because we will need the details of the construction.

Definition 1.29. - We denote by $\mathrm{PDE}_{L}$ the family of partial differential equations arising in Proposition 1.28.

Example 1.30. - If $L$ is the tautological relation of Example 1.6, then $\mathrm{PDE}_{L}$ is the following family of partial differential equations indexed by $d \geq 0$ and $\rho \in A$ :

$$
\frac{\partial F_{1}}{\partial t_{d+1}^{\rho}}-\sum_{\mu, \nu} \frac{\partial F_{1}}{\partial t_{0}^{\mu}} \eta^{\mu \nu} \frac{\partial^{2} F_{0}}{\partial t_{0}^{\nu} d t_{d}^{\rho}}-\frac{1}{24} \sum_{\mu, \nu} \frac{\partial^{3} F_{0}}{\partial t_{0}^{\mu} \partial t_{0}^{\nu} d t_{d}^{\rho}} \eta^{\mu \nu}=0 .
$$

Definition 1.31. - The linear combination $L \in G_{g, n}$ of stable dual graphs is called a universal relation if all formal Gromov-Witten potentials (Definition 1.23) satisfy the equations of the family $\mathrm{PDE}_{L}$.

The notion of a universal relation was introduced by Y.-P. Lee [24, 25]. 


\subsection{Main results}

1.5.1. Tautological, Gorenstein, and universal relations. - Recall that a linear combination $L \in G_{g, n}$ of dual graphs is a tautological relation if $[L]=0$, is a Gorenstein relation if the intersections of $[L]$ with tautological classes of complementary dimension vanish, and is a universal relation if all formal Gromov-Witten potentials satisfy $\mathrm{PDE}_{L}$.

Denote by Taut, Gor, and Univ the sets of tautological, Gorenstein, and universal relations respectively.

Theorem 1. - Gor $\subset$ Univ.

The proof of this theorem relies on Y.-P. Lee's work [25]. In his attempts to describe the set Univ, Y.-P. Lee constructs an algorithm that determines a subspace of $G_{d, n}$ and proves that this subspace is included in Univ. Here (Section 2.3) we give a geometric interpretation of this algorithm and show that the subspace it describes is actually nothing but Gor.

From the obvious inclusion Taut $\subset$ Gor we deduce

Corollary 1.32. - Taut $\subset$ Univ.

This corollary was also stated as a theorem by Y.-P. Lee, although his proof is quite complicated and involves a reference to C. Teleman's results.

Y.-P. Lee also formulated a conjecture that we can reformulate as

Conjecture 1.33. - Taut $=$ Gor $=$ Univ.

The inclusion Gor $\subset$ Taut is equivalent to the Gorenstein conjecture. The difficulties in establishing the inclusion Univ $\subset$ Gor are explained in Section 4.

1.5.2. Genus expansion. - Consider a power series $F=\sum \hbar^{g-1} F_{g} \in \hbar^{-1} \mathbb{C}\left[\left[\hbar ; t_{d}^{\mu}\right]\right]$, where $F_{0}$ is a formal genus 0 Gromov-Witten potential. We introduce five properties of $F$.

1. Homogeneity. $F$ is homogeneous in the sense of Definition 1.26.

2. Geometricity. $F$ satisfies all equations $\mathrm{PDE}_{L}$ for every tautological relation $L$.

3. Semi-simplicity. $F_{0}$ is semi-simple.

4. Reducibility to genus 0 . Every coefficient of $F$ can be expressed in terms of coefficients of $F_{0}$ using only properties 1 and 2 .

5. Analyticity. The homogeneous genus expansion of $F_{0}$ is regular at the origin.

THEOREM 2. - A power series F satisfying conditions (1-5) coincides with the homogeneous genus expansion of $F_{0}$.

All geometric Gromov-Witten potentials of target Kähler manifolds satisfy conditions 1 and 2. Conditions 3, 4, and 5, on the other hand, must be checked in every particular case.

As we show below, the series $F^{[r]}$ from Section 1.3.3 satisfies all five conditions (without being the geometric potential of a target manifold).

$4^{\mathrm{e}}$ SÉRIE - TOME $43-2010-\mathrm{N}^{\mathrm{o}} 4$ 


\subsubsection{Witten's $r$-spin conjecture}

THEOREM 3. - Witten's $r$-spin conjecture is true.

This theorem is the main application of Theorem 2. Let us outline the plan of the proof. The ideas we use are close to those used by Y.-P. Lee to prove Witten's conjecture in low genus in $[23,26]$.

In his initial paper E. Witten [35] proved that the conjecture was true in genus 0 provided the class $c_{W}$ satisfied certain postulated properties. The space of $r$-spin structures and the class $c_{W}$ were later rigorously constructed and shown to possess the expected properties by a joint effort of several people [16, 19, 28, 30, 31].

A. Givental [11] studied the homogeneous genus expansion of $F_{0}^{[r]}$ and showed that it was indeed a solution of the $r-\mathrm{KdV}$ hierarchy. Thus Witten's conjecture is reduced to the question: does $F^{[r]}$ coincide with its homogeneous genus expansion?

This is what we prove in this paper by checking that $F^{[r]}$ satisfies all the conditions of Theorem 2.

\section{Y.-P. Lee's algorithm and its geometrical interpretation}

\subsection{Preliminaries}

2.1.1. More on $\kappa$-classes. - Recall that

$$
\kappa_{k_{1}, \ldots, k_{m}}=\pi_{*}\left(\psi_{n+1}^{k_{1}+1} \ldots \psi_{n+m}^{k_{m}+1}\right) \text {, }
$$

where $\pi: \overline{\mathcal{M}}_{g, n+m} \rightarrow \overline{\mathcal{M}}_{g, n}$ is the forgetful map.

This definition is compatible with the usual definition of $\kappa$-classes $\kappa_{k}$ (for $m=1$ ). The classes $\kappa_{k_{1}, \ldots, k_{m}}$ and the monomials $\kappa_{k_{1}} \ldots \kappa_{k_{m}}$ form two bases of the same vector space and the matrix of basis change is triangular. Indeed, we have

$$
\kappa_{k_{1}, \ldots, k_{m}}=\sum_{\sigma \in S_{m}} \prod_{c=\text { cycle of } \sigma} \kappa_{k(c)}, \quad \text { where } \quad k(c)=\sum_{i \in c} k_{i} .
$$

For instance,

$$
\begin{aligned}
\kappa_{k_{1}, k_{2}} & =\kappa_{k_{1}} \kappa_{k_{2}}+\kappa_{k_{1}+k_{2}}, \\
\kappa_{k_{1}, k_{2}, k_{3}} & =\kappa_{k_{1}} \kappa_{k_{2}} \kappa_{k_{3}}+\kappa_{k_{1}+k_{2}} \kappa_{k_{3}}+\kappa_{k_{1}+k_{3}} \kappa_{k_{2}}+\kappa_{k_{2}+k_{3}} \kappa_{k_{1}}+2 \kappa_{k_{1}+k_{2}+k_{3}} .
\end{aligned}
$$

We prefer to work with the classes $\kappa_{k_{1}, \ldots, k_{m}}$ because they are easier to express in terms of Gromov-Witten correlators.

Let $p: \bar{M}_{g, n+1} \rightarrow \bar{M}_{g, n}$ be the forgetful map. Let $r: \bar{M}_{g-1, n+2} \rightarrow \bar{M}_{g, n}$ and $q: \overline{\mathcal{M}}_{g_{1}, n_{1}+1} \times \overline{\mathcal{M}}_{g_{2}, n_{2}+1} \rightarrow \overline{\mathcal{M}}_{g, n}$ be the usual "gluing" mappings to the boundary components of $\bar{M}_{g, n}$.

Lemma 2.1. - We have

$$
\begin{aligned}
p^{*}\left(\kappa_{k_{1}, \ldots, k_{m}}\right) & =\kappa_{k_{1}, \ldots, k_{m}}-\sum_{i=1}^{m} \psi_{n+1}^{k_{i}} \kappa_{k_{1}, \ldots, \hat{k}_{i}, \ldots, k_{m}}, \\
q^{*}\left(\kappa_{k_{1}, \ldots, k_{m}}\right) & =\sum_{I \sqcup J=\{1, \ldots, m\}} \kappa_{k_{I}} \times \kappa_{k_{J}}, \\
r^{*}\left(\kappa_{k_{1}, \ldots, k_{m}}\right) & =\kappa_{k_{1}, \ldots, k_{m}},
\end{aligned}
$$


where $k_{I}=\left\{k_{i}\right\}_{i \in I}, k_{J}=\left\{k_{i}\right\}_{i \in J}$, and $\widehat{k_{i}}$ means that the index is omitted.

Proof. - Only the first equality is nontrivial. Consider the forgetful map

$$
\tilde{p}: \overline{\mathcal{M}}_{g, n+1+m} \rightarrow \overline{\mathcal{M}}_{g, n+m} .
$$

To avoid confusion in indices, suppose the $n+m$ marked points are numbered from 1 to $n+m$, while the forgotten point is labeled with $\alpha$. In $H^{2}\left(\bar{M}_{g, n+m+1}\right)$ we have $\widetilde{p}^{*}\left(\psi_{n+i}\right)=$ $\psi_{n+i}-D_{n+i, \alpha}$, where $D_{n+i, \alpha}$ is the divisor of curves on which the points $n+i$ and $\alpha$ lie on a separate sphere with no other marked points. From the relations

$$
\psi_{n+i} D_{n+i, \alpha}=0 \quad \text { for } \quad 1 \leq i \leq m, \quad D_{n+i, \alpha} D_{n+j, \alpha}=0 \quad \text { for } i \neq j,
$$

we obtain that

$$
\widetilde{p}^{*}\left(\prod_{i=1}^{m} \psi_{n+i}^{k_{i}+1}\right)=\prod_{i=1}^{m} \psi_{n+i}^{k_{i}+1}+\sum_{i=1}^{m} \psi_{n+1}^{k_{1}+1} \ldots\left(-D_{n+i, \alpha}\right)^{k_{i}+1} \ldots \psi_{n+m}^{k_{m}+1} .
$$

Taking the push-forward of this class to $\bar{M}_{g, n+1}$ we obtain the right-hand side of the first equality of the lemma.

The second equality comes from the fact that each of the $m$ points forgotten by the map $\pi: \bar{M}_{g, n+m} \rightarrow \bar{M}_{g, n}$ can find itself on either of the two components of the boundary curves. The third equality follows directly from the definition.

2.1.2. More on dual graphs. - There are two natural generalizations of the notion of a stable dual graph.

First of all, we can consider not necessarily connected dual graphs. They represent tautological classes on direct products of several moduli spaces.

Second, we can consider dual graphs that describe the topology of a semi-stable curve that is not necessarily stable. Such graphs may have vertices of genus 0 with valency 2 . In this case we will also label each tail of the graph with a primary field $\mu \in A$. If we work over a Novikov ring, we also assign an element $D$ of the ring to the whole graph. Such a graph will be called a semi-stable dual graph with primary fields. It describes a tautological cohomology class in the space of stable maps $\bar{M}_{g, n+n^{\prime}, D}(X)$.

A "dual graph" with no other specifications will still mean "stable connected dual graph".

2.1.3. Constructing $\mathrm{PDE}_{L}$. - Let $F=\sum \hbar^{g-1} F_{g}$ be the geometric Gromov-Witten potential of some target Kähler manifold $X, A$ a basis of $H^{*}(X)$ and $\eta$ the Poincaré pairing. Let $L=\sum c_{i} G_{i}$ be a linear combination of dual graphs representing a class $[L] \in H^{*}\left(\overline{\mathcal{M}}_{g, n}\right)$. As in the introduction, let $X_{g, n+n^{\prime}, D}=\bar{M}_{g, n+n^{\prime}, D}(X)$ and let $\left[X_{g, n+n^{\prime}, D}\right]$ be its virtual fundamental class, and let $p: X_{g, n+n^{\prime}, D} \rightarrow \bar{M}_{g, n}$ be the forgetful map. We are going to describe a way to express the integrals of the form

$$
\sum_{D \in E} Q^{D} \int_{\left[X_{g, n+n^{\prime}, D}\right]} p^{*}([L]) \prod_{i=1}^{n+n^{\prime}} \psi_{i}^{d_{i}} \mathrm{ev}_{i}^{*}\left(\mu_{i}\right)
$$

via $\eta^{\mu \nu}$ and the coefficients of the series $F_{0}, \ldots, F_{g}$.

We start with assigning a polynomial in correlators and coefficients $\eta^{\mu \nu}$ to any stable dual graph or any semi-stable dual graph with primary fields that does not contain $\kappa$-classes. 
Definition 2.2. - Let $G$ be a stable dual graph or a semi-stable dual graph with primary fields such that no $\kappa$-classes are assigned to its vertices. We define the polynomial $P_{G}$ by the following procedure. (i) Assign a primary field $\mu \in A$ to every half-edge of $G$. In the case of a stable dual graph, assign, moreover, the distinguished primary field $1 \in A$ to the tails. (ii) To every vertex $v$ assign the correlator $\left\langle\tau_{d_{1}, \mu_{1}} \ldots \tau_{d_{n_{v}}, \mu_{n_{v}}}\right\rangle_{g_{v}}$, where $g_{v}$ is the genus of $v, n_{v}$ its valency, and $d_{i}, \mu_{i}$ the labels on the half-edges and tails adjacent to $v$. (iii) To every edge assign the coefficient $\eta^{\mu \nu}$, where $\mu$ and $\nu$ are the primary fields corresponding to its half-edges. (iv) Take the product of all the correlators and the coefficients $\eta^{\mu \nu}$ thus obtained. (v) Sum over all the ways to attribute primary fields to the half-edges. If we work over a Novikov ring, we must also sum over all the ways to assign effective 2-cycles $D_{v}$ to the vertices in such a way that $\sum D_{v}=D$.

Note that every edge of $G$ introduces a contraction of indices via the bilinear form $\eta$. This comes from the fact that the class of the diagonal in $X \times X$ equals $\sum_{\mu, \nu} \eta^{\mu \nu} \mu \times \nu$.

Note also that the definition works perfectly well for not necessarily connected dual graphs.

Now we go back to our problem of constructing a pull-back in $X_{g, n+n^{\prime}, D}$ of the tautological class $[L]$. Let $G$ be a stable dual graph participating in the linear combination $L$.

Step 1: eliminating the $\kappa$-classes.- If a vertex $v$ of $G$ is labeled with $\kappa_{k_{1}, \ldots, k_{m}}$, erase this label and replace it by $m$ new tails issuing from $v$ with labels $\psi^{k_{1}+1}, \ldots, \psi^{k_{m}+1}$ on them. Thus we obtain a new dual graph $G_{1}$. The newly added tails will be called $\kappa$-tails.

This rule is justified by the following remark: if $\pi: \bar{M}_{g, n+m} \rightarrow \overline{\mathcal{M}}_{g, n}$ is the forgetful map, we have

in $H^{*}\left(\overline{\mathcal{M}}_{g, n}\right)$.

$$
\psi_{1}^{d_{1}} \ldots \psi_{n}^{d_{n}} \kappa_{k_{1}, \ldots, k_{m}}=\pi_{*}\left(\psi_{1}^{d_{1}} \ldots \psi_{n}^{d_{n}} \psi_{n+1}^{k_{1}+1} \ldots \psi_{n+m}^{k_{m}+1}\right)
$$

EXAMPLE 2.3. - Let

$$
G=\begin{array}{cccc}
\psi^{0} \quad \kappa_{1} \quad \psi^{0} & \psi^{1} \\
g=2 & \bullet=1
\end{array}
$$

(here $g=3, n=1$ ). Then we have

$$
P_{G_{1}}=\sum_{\mu, \nu \in A}\left\langle\tau_{0,1} \tau_{2,1} \tau_{0, \nu}\right\rangle_{2} \eta^{\nu \mu}\left\langle\tau_{1, \mu}\right\rangle_{1}
$$

Step 2: recomputing the $\psi$-classes.- There is a difference between the $\psi$-classes on $\bar{M}_{g, n}$ and on $X_{g, n+n^{\prime}, D}$, because of the presence of additional marked points and because of the appearance of semi-stable source curves. To take this into account, we modify $G_{1}$ according to the following rule: replace every half-edge and every tail with label $\psi^{d}$

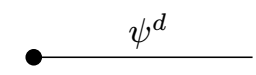

by the linear combination

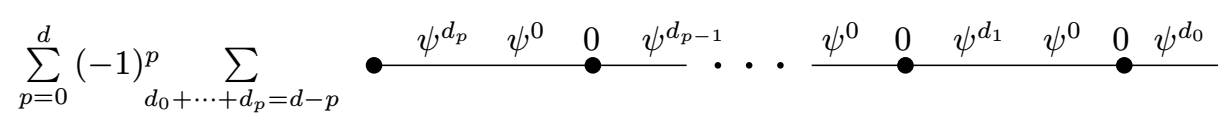


Thus we obtain a linear combination of dual graphs $G_{2}$. When we perform Steps 1 and 2 with all graphs of the linear combination $L$, we obtain a new linear combination $L_{2}$ of semistable dual graphs (without primary fields). It represents the class $p^{*}([L])$ and will therefore be denoted by $L_{2}=p^{*}(L)$.

In terms of polynomials $P_{G}$ assigned to the dual graphs, replacing $G_{1}$ by the linear combination of dual graphs $G_{2}$ is equivalent to making the following substitutions in $P_{G_{1}}$ :

$$
\begin{aligned}
\tau_{1, \mu} \mapsto & \tau_{1, \mu}-\sum_{\mu_{1}, \nu_{1}}\left\langle\tau_{0, \mu} \tau_{0, \nu_{1}}\right\rangle_{0} \eta^{\nu_{1} \mu_{1}} \tau_{0, \mu_{1}}, \\
\tau_{2, \mu} \mapsto & \tau_{2, \mu}-\sum_{\mu_{1}, \nu_{1}}\left\langle\tau_{1, \mu} \tau_{0, \nu_{1}}\right\rangle_{0} \eta^{\nu_{1} \mu_{1}} \tau_{0, \mu_{1}}-\sum_{\mu_{1}, \nu_{1}}\left\langle\tau_{0, \mu} \tau_{0, \nu_{1}}\right\rangle_{0} \eta^{\nu_{1} \mu_{1}} \tau_{1, \mu_{1}} \\
& +\sum_{\mu_{1}, \nu_{1}, \mu_{2}, \nu_{2}}\left\langle\tau_{0, \mu} \tau_{0, \nu_{1}}\right\rangle_{0} \eta^{\nu_{1} \mu_{1}}\left\langle\tau_{0, \mu_{1}} \tau_{0, \nu_{2}}\right\rangle_{0} \eta^{\nu_{2} \mu_{2}} \tau_{0, \mu_{2}},
\end{aligned}
$$

and so on. In general, every insertion of $\tau_{d, \mu}$ must be replaced by

$$
\tau_{d, \mu}+\sum_{p=1}^{d}(-1)^{p} \sum_{\substack{d_{0}+\cdots+d_{p}=d-p \\ \mu_{1}, \nu_{1}, \ldots, \mu_{p}, \nu_{p}}}\left\langle\tau_{d_{0}, \mu} \tau_{0, \nu_{1}}\right\rangle_{0} \eta^{\nu_{1} \mu_{1}}\left\langle\tau_{d_{1}, \mu_{1}} \tau_{0, \nu_{2}}\right\rangle_{0} \eta^{\nu_{2} \mu_{2}} \ldots \eta^{\nu_{p} \mu_{p}} \tau_{d_{p}, \mu_{p}} .
$$

This formula is used in the following way: the symbol $\tau_{d, \mu}$ was part of some correlator $\langle x\rangle$; now we put $\tau_{d_{p}, \mu_{p}}$ in its place, while the other factors of the formula become factors in front of $\langle x\rangle$.

It is important to note that the procedure of Step 2 for expressing ancestor $\psi$-classes in terms of descendant $\psi$-classes is universal. This means that the expression remains valid for any number of additional marked points $n^{\prime}$ and for any target manifold $X$.

Step 3: multiplying by $\prod_{i=1}^{n+n^{\prime}} \psi_{i}^{d_{i}} \mathrm{ev}_{i}^{*}\left(\mu_{i}\right)$.- Let $G_{2}$ be one of the dual graphs involved in the linear combination $L_{2}$. It has two kinds of tails: the $\kappa$-tails and the ordinary tails numbered from 1 to $n$. Add $n^{\prime}$ more tails numbered from $n+1$ to $n+n^{\prime}$ by attaching them to the vertices of $G_{2}$ in all possible ways.

Now, the $\kappa$-tails already bear labels $\psi^{k}$. We also label them with the distinguished primary field 1.

The tails from 1 to $n$ also bear labels $\psi^{d_{i}^{\prime}}, 1 \leq i \leq n$. We replace $\psi^{d_{i}^{\prime}}$ on the $i$-th tail by $\psi^{d_{i}^{\prime}+d_{i}}$ and also label the $i$-th tail with the primary field $\mu_{i}$.

The tails from $n+1$ to $n+n^{\prime}$ have no labels. We label them with $\psi^{d_{i}}$ and $\mu_{i}$, $n+1 \leq i \leq n+n^{\prime}$.

Thus we obtain a linear combination $L_{3}$ of semi-stable dual graphs with primary fields.

In terms of polynomials $P_{G}$, replacing $P_{L_{2}}$ by $P_{L_{3}}$ is equivalent to (i) replacing the symbols $\tau_{d_{i}^{\prime}, 1}$ corresponding to the tails from 1 to $n$ by symbols $\tau_{d_{i}^{\prime}+d_{i}, \mu_{i}}$ and (ii) inserting new symbols $\tau_{d_{i}, \mu_{i}}$ for $n+1 \leq i \leq n+n^{\prime}$ in the existing correlators in all possible ways.

Definition 2.4. - The linear combination of semi-stable dual graphs obtained by applying Steps 1, 2, and 3 to a linear combination of stable dual graphs $L \in G_{g, n}$ is denoted by $p^{*}(L) \prod_{i=1}^{n+n^{\prime}} \psi_{i}^{d_{i}} \operatorname{ev}_{i}^{*}\left(\mu_{i}\right)$. 
The corresponding polynomial in correlators is denoted by

$$
\left\langle p^{*}(L) \prod_{i=1}^{n+n^{\prime}} \psi_{i}^{d_{i}} \operatorname{ev}_{i}^{*}\left(\mu_{i}\right)\right\rangle_{g} .
$$

The geometric motivation of the three steps implies the following proposition.

Proposition 2.5. - If $F$ is the geometric Gromov-Witten potential of some target space $X$, we have

$$
\left\langle p^{*}(L) \prod_{i=1}^{n+n^{\prime}} \psi_{i}^{d_{i}} \mathrm{ev}_{i}^{*}\left(\mu_{i}\right)\right\rangle_{g}=\sum_{D \in H_{2}(X)} \int_{\left[X_{g, n+n^{\prime}, D}\right]} p^{*}([L]) \prod_{i=1}^{n+n^{\prime}} \psi_{i}^{d_{i}} \mathrm{ev}_{i}^{*}\left(\mu_{i}\right) .
$$

In particular, if $L$ is a tautological relation and $F$ is the geometric Gromov-Witten potential of some target space $X$, then

$$
\left\langle p^{*}(L) \prod_{i=1}^{n+n^{\prime}} \psi_{i}^{d_{i}} \operatorname{ev}_{i}^{*}\left(\mu_{i}\right)\right\rangle_{g}=0 .
$$

Note however that, for the time being, it is not at all clear whether the above expression vanishes for a formal Gromov-Witten potential.

Definition 2.6. - Let $L$ be a linear combination of dual graphs. Let $F=\sum \hbar^{g-1} F_{g}$ be a power series in variables $\hbar^{ \pm 1}$ and $t_{d}^{\mu}$. The infinite vector of the values of $\left\langle p^{*}(L) \prod_{i=1}^{n+n^{\prime}} \psi_{i}^{d_{i}} \operatorname{ev}_{i}^{*}\left(\mu_{i}\right)\right\rangle_{g}$ for all $n^{\prime} \geq 0$ and for all $d_{i}, \mu_{i}$, is called the induced vector of $L$ and is denoted by $F_{L}$.

Finally, let us explain the construction of the family of partial differential equations $\operatorname{PDE}_{L}$ from Proposition 1.28.

Sketch of proof of Proposition 1.28. - Let $L \in G_{g, n}$ be a linear combination of stable dual graphs. The family $\mathrm{PDE}_{L}$ is indexed by a list of integers $d_{1}, \ldots, d_{n} \geq 0$ and a list $\mu_{1}, \ldots, \mu_{n} \in A$. Given two lists like that we assign to them a partial differential equation in the following way. Consider the polynomial in correlators

$$
P=\left\langle p^{*}(L) \prod_{i=1}^{n} \psi_{i}^{d_{i}} \mathrm{ev}_{i}^{*}\left(\mu_{i}\right)\right\rangle_{g}
$$

(note that $n^{\prime}=0$ ). In this polynomial replace every correlator $\left\langle\tau_{\delta_{1}, \nu_{1}} \cdots \tau_{\delta_{p}, \nu_{p}}\right\rangle_{\gamma}$ by the partial derivative

$$
\frac{\partial^{p} F_{\gamma}}{\partial t_{\delta_{1}}^{\nu_{1}} \cdots \partial t_{\delta_{p}}^{\nu_{p}}}
$$

We have obtained a partial differential equation $\widehat{P}$.

The family of these differential equations is denoted by $\mathrm{PDE}_{L}$. It is easy to see that power series $F_{0}, \ldots, F_{g}$ satisfy all equations from $\mathrm{PDE}_{L}$ if and only if the induced vector $F_{L}$ vanishes. Indeed, the vanishing of

$$
\left\langle p^{*}(L) \prod_{i=1}^{n+n^{\prime}} \psi_{i}^{d_{i}} \mathrm{ev}_{i}^{*}\left(\mu_{i}\right)\right\rangle_{g}
$$


is equivalent to the vanishing of the coefficient of

$$
t_{d_{n+1}}^{\mu_{n+1}} \cdots t_{d_{n+n^{\prime}}}^{\mu_{n+n^{\prime}}}
$$

in $\widehat{P}$.

2.1.4. More on universal relations. - The notion of universal relation can be generalized to not necessarily connected stable dual graphs.

Consider any graph as a product of its connected components. Then a linear combination $L$ of not necessarily connected stable dual graphs is called a universal relation if it can be represented as a sum of several products such that every term of the sum contains a universal relation for connected graphs as a factor.

In other words, if we consider the product $\bar{M}_{g_{1}, n_{1}} \times \cdots \times \bar{M}_{g_{k}, n_{k}}$, a universal relation in one of the factors times any linear combination of graphs in the product of the remaining factors is a universal relation, and a linear combination of universal relations is still a universal relation.

REMARK 2.7. - If $F$ is the Gromov-Witten potential of a target manifold, then we can define the induced vector $F_{L}$ for a linear combination $L$ of not necessarily connected stable dual graphs in the same way as before. However, in this case a more natural notion is that of an extended induced vector $\widehat{F}_{L}$. Its definition is similar to that of $F_{L}$, with the difference that when we add new marked points to the curve, we are allowed to prescribe the connected component to which every point should go. If $L$ is a universal relation, then not only $F_{L}$, but also $\widehat{F}_{L}$ vanishes. However, only the vanishing of $F_{L}$ is encoded by the partial differential equations $\mathrm{PDE}_{L}$. This is why we cannot simply copy the definition of a universal relation to apply it to disconnected graphs.

In [25], Y.-P. Lee constructed an algorithm that computes certain, conjecturally all, universal relations. We are now going to describe this algorithm.

\subsection{Y.-P. Lee's algorithm}

2.2.1. The operators $\tau_{k}$. - We are going to define linear operators $\tau_{k}$ acting on the space of linear combinations of dual graphs. Here $k$ is an arbitrary positive integer.

If $G$ is a connected dual graph whose tails are labeled by a set $S$, then $\tau_{k}(G)$ is a linear combination of not necessarily connected dual graphs with labeling set $S \cup\{\alpha, \beta\}$. The graphs of this linear combination are obtained from $G$ by the following operations.

1. Cut an edge of $G$ into two tails. Change their labels to $\alpha$ and $\beta$ in both possible ways. If the labels on the half-edges were $\psi^{a}$ (on $\alpha$ ) and $\psi^{b}$ (on $\beta$ ) before the cutting, we now label them first with $\psi^{a+k}$ and $\psi^{b}$ and then with $\psi^{a}$ and $\psi^{b+k}$. The first stable graph thus obtained is taken with coefficient 1 while the second is taken with coefficient $(-1)^{k-1}$.

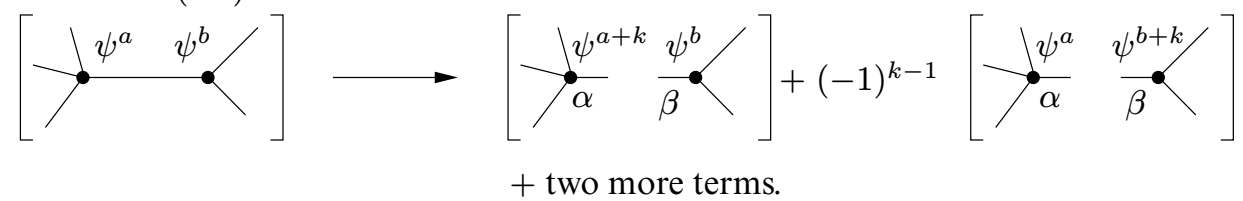

$4^{\text {e }}$ SÉRIE - TOME $43-2010-\mathrm{N}^{\mathrm{o}} 4$ 
2. Split a vertex $v$ of $G$ in two, and add a new tail on each of them, one marked by $\alpha$ and the other one by $\beta$. If the genus of $v$ was $g$, assign to the new vertices genera $g_{1}$ and $g_{2}$ such that $g_{1}+g_{2}=g$ in all possible ways. Distribute the edges that were going out of $v$ between the two vertices in all possible ways. If $v$ carried the label $\kappa_{k_{1}, \ldots, k_{m}}$, split the set $\left\{k_{1}, \ldots, k_{m}\right\}$ in two disjoint subsets $I$ and $J$ in all possible ways and assign to the new vertices the labels $\kappa_{I}$ and $\kappa_{J}$. Label tail $\alpha$ with $\psi^{i}$ and tail $\beta$ with $\psi^{j}$, in all possible ways with the condition $i+j=k-1$. The dual graph thus obtained is taken with coefficient $(-1)^{j+1}$. Keep only stable graphs and sum over all the possibilities described above.

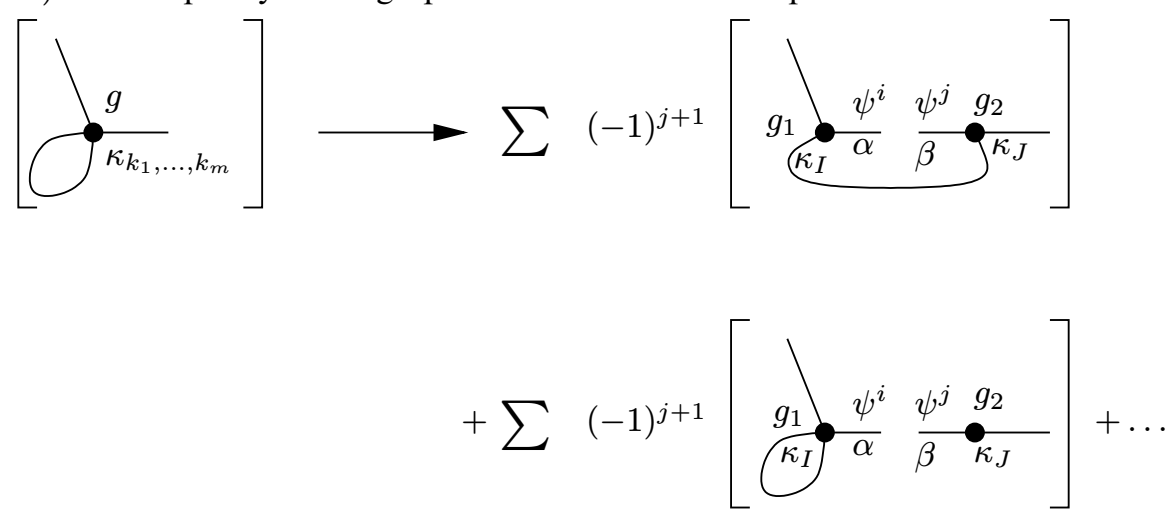

3. Choose a vertex of $G$, decrease its genus by 1 and add two new tails on it, one marked by $\alpha$ and the other one by $\beta$. Label tail $\alpha$ with $\psi^{i}$ and tail $\beta$ with $\psi^{j}$, in all possible ways with the condition $i+j=k-1$. The dual graph thus obtained is taken with coefficient $(-1)^{j+1}$. Sum over all possible $i$ and $j$.

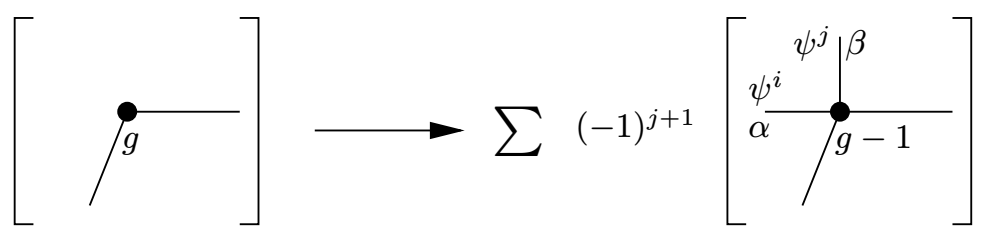

The operations $\tau_{k}$ are extended to linear combinations of dual graphs by linearity.

\subsubsection{The algorithm}

THEOREM 4 (Y.-P. Lee). - A linear combination L of dual graphs is a universal relation whenever (i) $F_{L}^{\mathrm{pt}}=0$ and (ii) $\tau_{k}(L)$ is a universal relation for all $k \geq 1$.

This theorem is actually an algorithm for computing universal relations for GromovWitten potentials. Indeed, the vector $F_{L}^{\mathrm{pt}}$ has an infinite number of entries, but they can be expressed via a finite number of entries using the string and dilaton equations. Therefore it is enough to check Condition (i) for a finite number of entries. Now, the dimension (see Definition 1.3) of $\tau_{k}(L)$ is smaller than that of $L$, therefore we can proceed by induction on the dimension of the relations.

Y.-P. Lee conjectures that this algorithm finds all universal relations on formal GromovWitten potentials and that these universal relations arise from tautological relations in the tautological ring of $\bar{M}_{g, n}$. However, neither of these claims is proved. 
Y.-P. Lee has also observed that in many cases Condition (i) is unnecessary and conjectured that checking Condition (ii) is enough for $\operatorname{dim} L \geq 1$. However, it follows from our geometric interpretation that this conjecture is wrong. The first example where it fails is in $\overline{\mathcal{M}}_{2,1}$ in dimension 1 .

Y.-P. Lee's proof of Theorem 4 is summarized in Section 4.

\subsection{The geometric interpretation}

In this section we give a geometric interpretation of the operators $\tau_{k}$ as intersections with the boundary. (This interpretation was discovered independently by Y.-P. Lee and R. Pandharipande - private communication.) Using it, we prove Theorem 1 and deduce from it Theorem 2.

2.3.1. Operators $\tau_{i}$ and boundary classes. - In the universal curve $\overline{\mathscr{C}}_{g, n}$, consider the codimension 2 subvariety $\Delta$ of nodes in the singular fibers of $\overline{\mathscr{C}}_{g, n}$. Each point of $\Delta$ is a node of a stable curve and we will label by $\alpha$ and $\beta$ the two marked points of its normalization identified at this node. This can be done in two ways, hence we obtain a double covering of $\Delta$ that we will call $\mathscr{D}$. The space $\mathscr{D}=\mathscr{D}_{g, n}$ has one connected component isomorphic to $\bar{M}_{g-1, n+2}$ (unless $g=0$ ) and $(g+1) 2^{n}-2(n+1)$ other connected components, each of which is isomorphic to $\bar{M}_{g_{1}, n_{1}+1} \times \bar{M}_{g_{2}, n_{2}+1}$ for suitable $g_{i}$ and $n_{i}$ with $g_{1}+g_{2}=g$ and $n_{1}+n_{2}=n$. It comes with a natural map $p: \mathscr{D} \rightarrow \overline{\mathcal{M}}_{g, n}$, whose image is the boundary $\partial \mathcal{M}_{g, n}=\overline{\mathcal{M}}_{g, n} \backslash \mathcal{M}_{g, n}$. Note that all tautological classes supported on $\partial \mathcal{M}_{g, n}$ are defined as push-forwards of classes on $\mathscr{D}$ under $p$.

On $\mathscr{D}$ we define the following cohomology classes:

$$
\begin{aligned}
& \rho_{1}=1 \\
& \rho_{2}=\psi_{\alpha}-\psi_{\beta} \\
& \rho_{3}=\psi_{\alpha}^{2}-\psi_{\alpha} \psi_{\beta}+\psi_{\beta}^{2} \\
& \rho_{4}=\psi_{\alpha}^{3}-\psi_{\alpha}^{2} \psi_{\beta}+\psi_{\alpha} \psi_{\beta}^{2}-\psi_{\beta}^{3}
\end{aligned}
$$

and so on.

Proposition 2.8. - Let $G$ be a dual graph of genus $g$ with $n$ tails. Then we have

$$
\left[\tau_{k}(G)\right]=-\rho_{k} p^{*}[G]
$$

in the cohomology ring of $\mathscr{D}$.

COROLlary 2.9. - Let $L \in G_{g, n}$ be a linear combination of dual graphs and suppose that the class $\left[\tau_{1}(L)\right]$ vanishes (respectively, has zero intersection with all tautological classes of complementary dimension). Then the class $\left[\tau_{k}(L)\right]$ vanishes (respectively, has zero intersection with all tautological classes of complementary dimension) for all $k$.

Proof. - The class $\left[\tau_{k}(L)\right]$ is obtained from $\left[\tau_{1}(L)\right]$ by a multiplication by $\rho_{k}$.

This corollary confirms Y.-P. Lee's experimental observation that requiring $\tau_{k}(L)$ to be a universal relation for all $k \geq 1$ is equivalent to requiring just $\tau_{1}(L)$ to be a universal relation ([24], Section 2.2, Remark (iii)).

$4^{\text {e }}$ SÉRIE - TOME $43-2010-\mathrm{N}^{\mathrm{o}} 4$ 
Proof of Proposition 2.8. - The main idea of the proof is very simple. On every boundary component of $\bar{M}_{g, n}$ we can define the classes $\psi_{\alpha}$ and $\psi_{\beta}$ corresponding to the marked points $\alpha$ and $\beta$ identified at the node. It is well-known that the first Chern class of the normal line bundle to the boundary component in $\bar{M}_{g, n}$ equals $-\left(\psi_{\alpha}+\psi_{\beta}\right)$.

Now, when we intersect a tautological class with a boundary component of $\bar{M}_{g, n}$ two cases can occur: either the class is entirely contained in the component, or it intersects it transversally. In the first case we must multiply our class by the first Chern class of the normal line bundle $-\left(\psi_{\alpha}+\psi_{\beta}\right)$ and then by $-\rho_{k}$. Their product is equal to $\psi_{\alpha}^{k}+(-1)^{k-1} \psi_{\beta}^{k}$. In the second case, we must add a new node, that either separates a component of the curve in two or is a nonseparating node. In both cases we multiply the class thus obtained by $-\rho_{k}=\sum_{i+j=k-1}(-1)^{j+1} \psi_{\alpha}^{i} \psi_{\beta}^{j}$. These three possibilities correspond to the three operations in the definition of $\tau_{k}$.

Now we present the proof with all necessary details.

To begin with, consider the case where $G$ is a dual graph of genus $g$ with $n$ tails with trivial labels assigned to all vertices, tails, and half-edges. Thus $[G]$ is the cohomology class $p_{G *}\left[\overline{\mathcal{M}}_{G}\right]$ on $\overline{\mathcal{M}}_{g, n}$.

The computation of $p^{*}[G]$ fits in the framework of [12], A.4. The contributing graphs are of two kinds: either they have one more edge than $G$ or they have the same number of edges as $G$.

Consider a contributing graph with one extra edge. Contracting the extra edge, we obtain the graph $G$ and the contracted edge determines a unique vertex $v$ of $G$; let $g_{v}$ be the geometric genus of the corresponding component and let $n_{v}$ be the valence of $G$ at $v$ including both half-edges and tails. Unless $g_{v}=0$, there is exactly one contributing graph corresponding to $v$ whose extra edge is a self-edge at a vertex with geometric genus $g_{v}-1$. Moreover, there are exactly $\left(g_{v}+1\right) 2^{n_{v}}-2\left(n_{v}+1\right)$ other contributing graphs corresponding to $v$ whose extra edge connects two distinct vertices. In other words, the contributing graphs whose extra edge contracts to $v$ are in one-to-one correspondence with the connected components of the double covering $\mathscr{D}_{g_{v}, n_{v}}$. Let $\Gamma$ be a contributing graph with extra edge $e$. Let $h_{\alpha}$ and $h_{\beta}$ be the labeled half-edges constituting $e$ and let $\alpha$ and $\beta$ be the corresponding tails arising upon cutting $e$. If cutting $e$ disconnects the graph, put

$$
\overline{\mathcal{M}}_{e}:=\overline{\mathcal{M}}_{g_{\alpha}, n_{\alpha}} \times \overline{\mathcal{M}}_{g_{\beta}, n_{\beta}}
$$

where $g_{\alpha}$ resp. $g_{\beta}$ and $n_{\alpha}$ resp. $n_{\beta}$ are the genus and the number of tails of the connected component containing $\alpha$ resp. $\beta$. If $e$ is non-disconnecting, put

$$
\bar{M}_{e}:=\bar{M}_{g-1, n+2} \text {. }
$$

Further, let

$$
p_{\Gamma, e}: \overline{\mathcal{M}}_{\Gamma} \rightarrow \overline{\mathcal{M}}_{e} \subset \mathscr{D}_{g, n}
$$

be the map obtained via cutting $e$ and contracting all other edges. Then by [12], A.4 the contribution of $(\Gamma, e)$ to $p^{*}[G]$ equals

$$
p_{\Gamma, e *}\left[\overline{\mathcal{M}}_{\Gamma}\right] .
$$


Next, consider a contributing graph with the same number of edges as $G$. The graph may then be identified with $G$ and exactly one edge $e$ of $G$ has been selected: it is identified by the excess line bundle. Define $\bar{M}_{e}$ as above and let

$$
p_{G, e}: \bar{M}_{G} \rightarrow \bar{M}_{e} \subset \mathscr{D}_{g, n}
$$

be the map obtained via cutting $e$ and contracting all other edges. Then by [12], A.4 the contribution of $(G, e)$ to $p^{*}[G]$ equals

$$
p_{G, e *}\left(-\psi_{\alpha}-\psi_{\beta}\right)=\left(-\psi_{\alpha}-\psi_{\beta}\right) p_{G, e *}\left[\bar{M}_{G}\right]
$$

(with a slight abuse of notation).

We find the following formula for $p^{*}[G]$ :

$$
p^{*}[G]=\sum_{(\Gamma, e): \Gamma / e=G} p_{\Gamma, e *}[\Gamma]+\sum_{e=\left(h_{\alpha}, h_{\beta}\right) \in E(G)}\left(-\psi_{\alpha}-\psi_{\beta}\right) p_{G, e *}[G] .
$$

If $v_{0}(G)$ denotes the number of vertices of $G$ of geometric genus 0 , the number of summands in the first sum equals

$$
\sum_{v \in V(G)}\left(\left(g_{v}+1\right) 2^{n_{v}}-2 n_{v}-2\right)+|V(G)|-v_{0}(G)
$$

while the second sum runs over the half-edges of $G$.

Having dealt with the case of a dual graph with trivial labels, we consider the case where $G$ is a decorated dual graph: each vertex $v$ of $G$ has been assigned a class $\kappa_{K_{v}}$, where $K_{v}$ is a collection of $m_{v}$ nonnegative integers, and each half-edge or tail $h$ has been assigned a power $\psi_{h}^{d_{h}}$ of its $\psi$-class $\psi_{h}$.

Each half-edge or tail on $G$ determines a unique such object on $\Gamma$. Taking this into account, the formula above for $p^{*}[G]$ continues to hold in the case of decorated half-edges or tails.

Let $v$ be a vertex of $G$. In case the extra edge $e$ on $\Gamma$ does not contract to $v$, a unique vertex $w$ of $\Gamma$ corresponds to $v$ and it is decorated with the corresponding class $\kappa_{K_{v}}$. We proceed analogously in case $e$ is a self-edge at a vertex $w$ (with genus $g_{w}=g_{v}-1$ ) contracting to $v$. Finally, if $e$ contracts to $v$ and connects two distinct vertices, the decoration $\kappa_{K_{v}}$ has to be divided up in all $2^{m_{v}}$ possible ways over the two vertices (cf. Lemma 2.1). The formula above for $p^{*}[G]$ continues to hold for an arbitrary decorated dual graph; note that the number of summands in the first sum equals

$$
\sum_{v \in V(G)} 2^{m_{v}}\left(\left(g_{v}+1\right) 2^{n_{v}}-2 n_{v}-2\right)+|V(G)|-v_{0}(G) .
$$

These considerations prove the statement for $\tau_{1}$. The statement for $\tau_{k}$ follows from the trivial remark that

$$
\rho_{k}\left(\psi_{\alpha}+\psi_{\beta}\right)=\psi_{\alpha}^{k}+(-1)^{k-1} \psi_{\beta}^{k}
$$

$4^{\mathrm{e}}$ SÉRIE - TOME $43-2010-\mathrm{N}^{\mathrm{o}} 4$ 


\subsubsection{The algorithm and the Gorenstein conjecture}

Theorem 5. - A linear combination L of stable dual graphs appears as a result of $Y$.-P. Lee's algorithm if and only if it is a Gorenstein relation.

Proof. - Denote by $p: \overline{\mathcal{M}}_{g, n+n^{\prime}} \rightarrow \overline{\mathcal{M}}_{g, n}$ the forgetful map and by $q: \mathscr{D} \rightarrow \overline{\mathcal{M}}_{g, n}$ the natural projection.

Taking into account the form of Y.-P. Lee's algorithm (Section 2.2.2), and the geometric interpretation of the operators $\tau_{k}$, the theorem can be reformulated as follows:

A tautological class $\alpha \in H^{*}\left(\overline{\mathcal{M}}_{g, n}\right)$ is Gorenstein vanishing if and only if

(i) all the intersection numbers

$$
\int_{\bar{M}_{g, n+n^{\prime}}} p^{*}(\alpha) \prod_{i=1}^{n+n^{\prime}} \psi_{i}^{d_{i}}
$$

vanish; and

(ii) the classes $\rho_{k} q^{*}(\alpha)$ are Gorenstein vanishing for all $k$.

Since $\rho_{1}=1$, the second condition can, of course, be replaced by the condition $q^{*}(\alpha)$ is Gorenstein vanishing.

The only if part. Suppose $\alpha$ is Gorenstein vanishing. Then

$$
\int_{\bar{M}_{g, n+n^{\prime}}} p^{*}(\alpha) \prod_{i=1}^{n+n^{\prime}} \psi_{i}^{d_{i}}=\int_{\bar{M}_{g, n}} \alpha p_{*}\left(\prod_{i=1}^{n+n^{\prime}} \psi_{i}^{d_{i}}\right)=0,
$$

because the class $p_{*}\left(\prod_{i=1}^{n+n^{\prime}} \psi_{i}^{d_{i}}\right)$ is tautological.

Similarly, if $\beta$ is a tautological class on $\mathscr{D}$, then

$$
\int_{\mathscr{D}} q^{*}(\alpha) \beta=\int_{\overline{\mathcal{M}}_{g, n}} \alpha q_{*}(\beta)=0,
$$

because $q_{*}(\beta)$ is tautological.

The if part. Suppose $\alpha$ satisfies Conditions (i) and (ii), and let $G$ be a dual graph of genus $g$ with $n$ tails, of complementary dimension to $\alpha$. We wish to prove that $\alpha \cap[G]=0$.

First consider the case when $G$ has no edges. This means that $[G]$ is a polynomial in $\psi$ and $\kappa$-classes. A class like that can be represented as a linear combination of several classes $p_{*}\left(\prod_{i=1}^{n+n^{\prime}} \psi_{i}^{d_{i}}\right)$. But, by the same equality that we used in the "if" part, we have

$$
\int_{\bar{M}_{g, n}} \alpha p_{*}\left(\prod_{i=1}^{n+n^{\prime}} \psi_{i}^{d_{i}}\right)=\int_{\bar{M}_{g, n+n^{\prime}}} p^{*}(\alpha) \prod_{i=1}^{n+n^{\prime}} \psi_{i}^{d_{i}}=0
$$

by Condition (i).

Now suppose $G$ has at least one edge. Then the class $[G]$ is the push-forward of some tautological class $\beta$ on $\mathscr{D}$, in other words, $[G]=q_{*}(\beta)$. Thus

$$
\alpha \cap[G]=\int_{\bar{M}_{g, n}} \alpha q_{*}(\beta)=\int_{\mathscr{D}} q^{*}(\alpha) \beta=0,
$$


because $q^{*}(\alpha)$ is Gorenstein vanishing by Condition (ii).

2.3.3. Proofs of Theorems 1 and 2. - Our first two main theorems are now almost immediate.

Proof of Theorem 1. - It follows from Theorems 4 and 5. Indeed, Theorem 5 says that a linear combination of dual graphs appears as a result of Y.-P. Lee's algorithm if and only if it is a Gorenstein relation. On the other hand, Theorem 4 says that every linear combination of dual graphs that appears as a result of Y.-P. Lee's algorithm is a universal relation.

Proof of Theorem 2. - Let $\widetilde{F}$ be the homogeneous genus expansion of $F_{0}$, which can be constructed by condition (3) (semi-simplicity). By condition (5) (analyticity), every $\widetilde{F}_{g}$ is a power series. Therefore the induced vector $\widetilde{F}_{L}$ makes sense, and the vanishing of $\operatorname{PDE}_{L}(\widetilde{F})$ is equivalent to the vanishing of $\widetilde{F}_{L}$. Both $F_{L}$ and $\widetilde{F}_{L}$ vanish for all tautological relations $L$. Indeed, $F_{L}$ vanishes by condition (2) (geometricity), while $\widetilde{F}_{L}$ vanishes by Theorem 1 . Similarly, the nonhomogeneous correlators vanish both for $F$ (by condition (1), homogeneity) and for $\widetilde{F}$ (because $\widetilde{F}$ is the homogeneous genus expansion). According to condition (4) (reducibility to genus 0 ), these vanishing conditions are enough to express every correlator in terms of genus 0 correlators. But the genus 0 correlators coincide, since both are given by $F_{0}$. Thus $F=\widetilde{F}$.

\section{A proof of Witten's conjecture}

In this section we explain in more detail the plan of the proof of Witten's conjecture outlined in Section 1.5.3.

\subsection{Givental's work}

In [10], Givental constructed the homogeneous genus expansion of $F_{0}^{[r]}$ and proved that it was indeed a solution of the $r-\mathrm{KdV}$ hierarchy. Let us denote this genus expansion by $F^{(r)}$.

Thus we have two power series: $F^{[r]}$ and $F^{(r)}$. The first one is the "geometric" GromovWitten potential: its coefficients are integrals of cohomology classes over $\overline{\mathcal{M}}_{g, n}$. The second one is a formal Gromov-Witten potential: it is the homogeneous genus expansion of $F_{0}^{[r]}$. It is also a solution of the $r-\mathrm{KdV}$ hierarchy. What remains to be proved is that $F^{[r]}=F^{(r)}$. To do that, we will use Theorem 2, so let us check the conditions (1-5) involved in its formulation.

Homogeneity. The correlator

vanishes unless

$$
\left\langle\tau_{d_{1}, a_{1}} \ldots \tau_{d_{n}, a_{n}}\right\rangle_{g}
$$

$$
\sum d_{i}+\sum \frac{a_{i}}{r}=n+\left(\frac{r-2}{r}-3\right)(1-g)
$$

Indeed, from the definitions of Section 1.3, we get $c_{1}(\mathcal{T})=\left(2 g-2-\sum a_{i}\right) / r$, hence the rank of $V$ and the degree of $c_{W}$ equal

$$
\operatorname{deg} c_{W}=\frac{(r-2)(g-1)+\sum a_{i}}{r} .
$$

Thus the series $F^{[r]}$ satisfies the homogeneity property for the weight function $w(i)=\frac{i}{r}-1$, and the "dimension" $\operatorname{dim}=\frac{r-2}{r}$. (There is no need for a weight function on a Novikov ring, because we are working over $\mathbb{C}$.)

$4^{\mathrm{e}}$ SÉRIE - TOME $43-2010-\mathrm{N}^{\mathrm{o}} 4$ 
Geometricity. It is obvious that the Gromov-Witten potential $F^{[r]}$ respects all tautological relations because of its geometric origin.

Semi-simplicity. The semi-simplicity of the Frobenius manifold assigned to $F_{0}^{[r]}$ is wellknown, see, for example, the introduction of [10]. To sum up, there exists a bi-polynomial isomorphism

$$
\mathbb{C}^{r-2} \rightarrow \mathbb{C}^{r-2}:\left(t_{0}^{1}, \ldots, t_{0}^{r-2}\right) \mapsto\left(s_{1}, \ldots, s_{r-2}\right)
$$

such that the algebra described in Definition 1.17 is naturally identified with the algebra

$$
\mathbb{C}[X] /\left(X^{r-1}+s_{1} X^{r-3}+\cdots+s_{r-2}\right) .
$$

Thus it is semi-simple whenever the polynomial has $r-1$ distinct roots.

For instance, for $r=5$, the algebra of Definition 1.17 is isomorphic to $\mathbb{C}[X] / P^{\prime}$, where

$$
P(X)=X^{5}-t_{0}^{3} X^{3}-t_{0}^{2} X^{2}+\left(\left(t_{0}^{3}\right)^{2} / 5-t_{0}^{1}\right) X
$$

and $P^{\prime}$ is its derivative.

Reducibility to genus 0 . This property will be established in Theorem 6.

Analyticity. In [10] the genus expansion of $F_{0}^{(r)}$ is given in the form of a power series at the origin.

The rest of this section is devoted to proving the reducibility to genus 0 .

\subsection{Admissible covers and double ramification cycles}

The spaces of admissible covers and the double ramification cycles were first introduced by Ionel [15] and proved very useful in the study of moduli spaces. Let us briefly recall their definitions.

Consider a map $\varphi$ from a smooth curve $C$ with $n$ marked points to the sphere $S=\mathbb{C P}^{1}$. On $S$ we mark all branch points of $\varphi$ and the images of the $n$ marked points of $C$. On $C$ we then mark all the preimages of the points that are marked on $S$. Now choose several disjoint simple loops on $S$, that do not pass through the marked points. Suppose that if we contract these loops we obtain a stable genus 0 curve $S^{\prime}$. Now contract also all the preimages of the loops in $C$ to obtain a nodal curve $C^{\prime}$ that turns out to be automatically stable. We have obtained a map $\varphi^{\prime}$ from a stable curve $C^{\prime}$ of genus $g$ to a stable curve $S^{\prime}$ of genus 0 . It has the same degree over every component of $S^{\prime}$. Moreover, at each node of $C^{\prime}$, the projection $\varphi^{\prime}$ has the same local multiplicity on both branches meeting at the node.

Definition 3.1. - A map from a stable curve of genus $g$ to a stable curve of genus 0 topologically equivalent to a map described above is called an admissible covering.

We will be particularly interested in the space of admissible coverings with multiple ramifications over only 2 points labeled with 0 and $\infty$, the other ramification points being simple.

Definition 3.2. - Consider the space of admissible coverings of some given genus $g$ with prescribed ramification types over two points labeled 0 and $\infty$, and with simple ramifications elsewhere. The normalization of this space is called a double ramification space or a DR-space. 
Definition 3.3. - Let $k_{1}, \ldots, k_{n+p}$ be a list of integers such that $\sum k_{i}=0$ and $k_{i} \neq 0$ for $n+1 \leq i \leq n+p$. Consider the set of smooth curves $\left(C, x_{1}, \ldots, x_{n}\right) \in \mathcal{M}_{g, n}$ such that there exist $p$ more marked points $x_{n+1}, \ldots, x_{n+p}$ and a meromorphic function on $C$ with no zeroes or poles outside of $x_{1}, \ldots, x_{n+p}$, the orders of zeroes or poles being prescribed by the list $k_{1}, \ldots, k_{n+p}\left(k_{i}>0\right.$ for the zeroes, $k_{i}<0$ for the poles, and $k_{i}=0$ for the marked points that are neither zeroes nor poles). The closure of this set in $\bar{M}_{g, n}$ is called the double ramification cycle or a $D R$-cycle.

Here are some basic facts about the double ramification cycles that make them so useful.

1. The codimension of a DR-cycle is equal to $g-p$ whenever there is at least one positive and one negative number among $k_{1}, \ldots, k_{n}$ (see [15,29]). Assuming that this condition is satisfied we see that for $p=g$ the DR-cycle coincides with the moduli space $\bar{M}_{g, n}$.

2. The cohomology class Poincaré dual to any DR-cycle belongs to the tautological ring of $\overline{\mathcal{M}}_{g, n}$ (proved in [7]).

This makes the results of this paper applicable to DR-cycles.

3. Each DR-cycle is the image of the corresponding DR-space under the forgetful map $\pi$ that forgets the covering and all the marked points except $x_{1}, \ldots, x_{n}$, but retains and stabilizes the source curve with the $n$ remaining marked points. The map $\pi$ sends the fundamental homology class of the DR-space to a multiple of the fundamental homology class of the DR-cycle.

4. Every class $\pi^{*}\left(\psi_{i}\right)$ on a DR-space can be expressed as a linear combination of boundary divisors $[15,32]$.

This is a very important property that can be used to compute integrals involving $\psi$-classes. Indeed, it allows us to get rid of the $\psi$-classes one by one by reducing the integral to simpler integrals over smaller spaces. Using this procedure, the following result was established in [34], Theorem 1.

\subsection{Reducibility to genus 0}

THEOREM 6 ([34]). - Every correlator in the r-spin Witten conjecture can be expressed in genus zero correlators using only tautological relations.

The last result shows that Theorem 2 is applicable to the $r$-spin Witten conjecture and suffices to prove it.

The proof of Theorem 6 in [34] goes as follows. As explained in Item 1, the fundamental class of $\bar{M}_{g, n}$ can be represented as a DR-cycle with $p=g$. If this is done in an intelligent way, then the elimination of $\psi$-classes according to Item 4 , leads us to boundary divisors that can themselves be expressed as DR-cycles on the boundary. We end up with the integral of Witten's class $c_{W}$ (without $\psi$-classes) over a DR-cycle. A dimension count shows that an integral like that may be nonzero only if $g=0$ or 1 and the codimension of the DR-cycle is equal to the genus ( 0 for $g=0$ and 1 for $g=1$ ). In the latter case we must do some more work: putting one $\psi$-class back into the integral and expressing it as a linear combination of boundary divisors in different ways we obtain certain relations between genus 1 and genus 0 integrals of $c_{W}$. It turns out that these relations suffice to reduce all genus 1 integrals to genus 0 integrals.

$4^{\mathrm{e}}$ SÉRIE - TOME $43-2010-\mathrm{N}^{\mathrm{o}} 4$ 
However, now we can give a simpler, although less constructive, proof.

Proof of Theorem 6. - In [15], Ionel proved the following assertion:

Let $M$ be a monomial in $\psi$-and $\kappa$-classes on $\overline{\mathcal{M}}_{g, n}$, of degree ${ }^{(2)}$ at least $g$ for $g \geq 1$ or at least 1 for $g=0$. Then the class $M$ can be represented as a linear combination of classes of the form

$$
q_{*}\left[(\mathrm{DR} \text {-cycle })_{1} \times \cdots \times(\mathrm{DR}-\text { cycle })_{k}\right] .
$$

Here $k \geq 1$ is an integer that can be different for different terms of the sum, $q: \bar{M}_{g_{1}, n_{1}} \times \cdots \times \overline{\mathcal{M}}_{g_{k}, n_{k}} \rightarrow \overline{\mathcal{M}}_{g, n}$ is the gluing map from a product of smaller moduli spaces to a boundary stratum of $\bar{M}_{g, n}$ and the cycles (DR-cycle) ${ }_{j}$ are DR-cycles on the smaller moduli spaces.

It was established in [7] that every DR-cycle is tautological and that Ionel's theorem can therefore be improved in the following way:

Let $M$ be a monomial in $\psi$ - and $\kappa$-classes on $\overline{\mathcal{M}}_{g, n}$, of degree at least $g$ for $g \geq 1$ or at least 1 for $g=0$. Then the class $M$ can be represented by a linear combination of dual graphs each of which has at least one edge.

We will call this property the $g$-reduction.

Now, a simple dimension count shows that the integral

$$
\int_{\bar{M}_{g, n}} \beta \cdot c_{W}\left(a_{1}, \ldots, a_{n}\right)
$$

vanishes unless the class $\beta$ has complex degree at least $g$. Indeed, the degree of Witten's class equals

$$
\operatorname{deg} c_{W}=\frac{(r-2)(g-1)+\sum a_{i}}{r} \leq \frac{(r-2)(n+g-1)}{r},
$$

while the dimension of $\overline{\mathcal{M}}_{g, n}$ is $3 g-3+n$ (use the exact expression for $g=1$ and the upper bound for $g \geq 2$ ).

Recall that the pull-back of Witten's class to the boundary components is given by the factorization property (3) of Section 1.3.2. This makes it easy to apply the $g$-reduction to integrals involving Witten's class.

The rest of the proof is simple. Suppose we wish to compute the integral

$$
\int_{\bar{M}_{g, n}} c_{W}\left(a_{1}, \ldots, a_{n}\right) \psi_{1}^{d_{1}} \ldots \psi_{n}^{d_{n}} .
$$

Apply the $g$-reduction in iteration as many times as possible, starting with the class $\psi_{1}^{d_{1}} \ldots \psi_{n}^{d_{n}}$. In the end we will obtain an expression of $\prod_{i=1}^{n} \psi_{i}^{d_{i}}$ as a linear combination of dual graphs $G$ satisfying the following condition. Suppose a vertex $v$ of $G$ is labeled with genus $g_{v}>0$ and with a class $\kappa_{k_{1}, \ldots, k_{m}}$, and suppose the half-edges and tails issuing from $v$ are labeled with $\psi^{d_{1}}, \ldots, \psi^{d_{n_{v}}}$. Then $\sum k_{i}+\sum d_{i}<g_{v}$. (Indeed, if $\sum k_{i}+\sum d_{i} \geq g_{v}$ for at least one vertex, we can apply the $g$-reduction to this vertex.) But, as we have already explained, the integral of Witten's class over the class $[G]$ represented by a dual graph like that vanishes whenever there is at least one vertex of nonzero genus. Thus the only contribution comes from graphs with only genus zero vertices with no $\psi$ - or $\kappa$-classes. So we

(2) The degree of a $\psi$-class equals 1 , while the degree of $\kappa_{k}$ equals $k$. 
have reduced any given correlator involved in Witten's conjecture to a linear combination of products of genus 0 correlators. More precisely, the only remaining correlators are integrals of Witten's class with no $\psi$-classes over genus zero moduli spaces.

This completes the proof of Theorem 6. As we have already explained, it implies that the formal Gromov-Witten potential $F^{(r)}$ coincides with the geometric Gromov-Witten potential $F^{[r]}$ and proves the Witten conjecture.

\section{More on Givental's quantization and Y.-P. Lee's theorem}

A proof of Theorem 4 is contained in [25]. However there are some missing details that we would like to fill in here. First, we would like to explain precisely why the operators $\tau_{k}$ act on the $\kappa$-classes in the way described in Section 2.2.1 (this is done in Proposition 4.5). Second, we explain more precisely how Givental's quantization is applied to prove the theorem.

"Givental's group" is really not a group, but a collection of two groups: the so-called "lower triangular" and "upper triangular" groups. This is analogous to the Birkhoff decomposition in the finite-dimensional case. However, because both groups are infinitedimensional, it is in general not possible to multiply their elements, like it is impossible to multiply a power series in $z$ and a power series in $z^{-1}$. On the other hand, it turns out that, under some conditions, one can apply first an element of the upper triangular group and then an element of the lower triangular group to a Gromov-Witten potential.

Both the lower triangular and the upper triangular group possess Lie algebras. An element of the lower triangular Lie algebra is a series $s\left(z^{-1}\right)=\sum_{l \geq 1} s_{l} z^{-l}$ of linear operators on the vector space $V$. The operators $s_{l}$ are self-adjoint for $l$ odd and skew-selfadjoint for $l$ even with respect to the quadratic form $\eta$.

Similarly, an element of the upper triangular Lie algebra is a series $r(z)=\sum_{l \geq 1} r_{l} z^{l}$ of linear operators on $V$. The operators $r_{l}$ are self-adjoint for $l$ odd and skew-self-adjoint for $l$ even with respect to the quadratic form $\eta$.

The notation $\left(r_{l}\right)_{\mu}^{\nu},\left(r_{l}\right)_{\mu \nu},\left(r_{l}\right)^{\mu \nu}$ and similarly for $s_{l}$ was introduced in Section 1.4.1.

Y.-P. Lee [25] writes down explicit formulas for the action of $s$ and $r$ on any given correlator of a Gromov-Witten potential $F$ (see below). Once this is done, the main problem is to understand what happens when we apply these formulas to the induced vector of a tautological class: indeed, both Y.-P. Lee's formulas for the derivatives of an individual correlator and the expression of the induced vector in terms of correlators (described in Section 2.1.3) are fairly complicated.

Below we sum up the argument of [25] and give more detailed statements of certain results.

If $s\left(z^{-1}\right)=\sum_{l \geq 1} s_{l} z^{-l}$ is an element of the lower triangular Lie algebra and $r(z)=\sum_{l \geq 1} r_{l} z^{l}$ an element of the upper triangular Lie algebra, denote, for shortness

$$
s_{l}\left(\tau_{d, \mu}\right)=\sum_{\nu}\left(s_{l}\right)_{\mu}^{\nu} \tau_{d-l, \nu}, \quad r_{l}\left(\tau_{d, \mu}\right)=\sum_{\nu}\left(r_{l}\right)_{\mu}^{\nu} \tau_{d+l, \nu}
$$

Now we are going to follow the path from the Gromov-Witten potential of a point to the general formal semi-simple Gromov-Witten potential.

$4^{\text {e }}$ SÉRIE - TOME $43-2010-\mathrm{N}^{\mathrm{o}} 4$ 


\subsection{The upper triangular group}

Let $t_{0}^{\mu}$ be flat coordinates on a semi-simple Frobenius manifold $M$ of dimension $k$. Let $f\left(t_{0}^{\mu}\right)$ be the corresponding genus 0 potential. We assume that $f$ is an analytic function. At a semi-simple point, the tangent Frobenius algebra $T_{*} M$ to the Frobenius manifold $M$ possesses a basis of primitive idempotents. Denote by $\alpha_{1}, \ldots, \alpha_{k}$ their scalar squares in the metric $\eta$. Givental constructs an element $R$ of the upper triangular group whose action transforms the constant Frobenius structure on $T_{*} M$ into the Frobenius structure of $M$ at the neighborhood of the semi-simple point.

In other words, the first step of Givental's quantization is to apply the quantized action of $R$ to the series $\exp \left(F^{\alpha_{1}, \ldots, \alpha_{k}}\right)$, see Equation (2) from Section 1.2.2.

The potential $F^{\alpha_{1}, \ldots, \alpha_{k}}$ obviously possesses the two following crucial properties.

Definition 4.1. - A Gromov-Witten potential is tame if $\left\langle\tau_{d_{1}}^{\mu_{1}} \cdots \tau_{d_{n}}^{\mu_{n}}\right\rangle_{g}$ vanishes whenever $\sum d_{i}>3 g-3+n$.

Definition 4.2. - A Gromov-Witten potential is an ancestor potential if its correlators with $2-2 g-n \geq 0$ vanish.

Let $r=\ln R$ be an element of the upper triangular Lie algebra. In general, the action of $R$ on a power series is not well defined. However, it is easy to check (cf. Proposition 4.3) that the action of $r_{l}$ increases the grading $\sum d_{i}-n-3 g+3$ by $l$. Therefore the action of $R$ on a tame series is well-defined and is equal to the exponential of the action of $r$.

Translating the action of the operator $\widehat{r}$ given in Section 1.4.1 in terms of individual coefficients we obtain the following result.

Proposition 4.3 ([25], Equation (23)). - We have

$$
\begin{aligned}
r . & \left\langle\tau_{d_{1}, \mu_{1}} \ldots \tau_{d_{n}, \mu_{n}}\right\rangle_{g}= \\
& -\sum_{l=1}^{\infty}\left\langle r_{l}\left(\tau_{1,1}\right) \tau_{d_{1}, \mu_{1}} \ldots \tau_{d_{n}, \mu_{n}}\right\rangle_{g} \\
& +\sum_{l=1}^{\infty} \sum_{i=1}^{n}\left\langle\tau_{d_{1}, \mu_{1}} \ldots r_{l}\left(\tau_{d_{i}, \mu_{i}}\right) \ldots \tau_{d_{n}, \mu_{n}}\right\rangle_{g} \\
& +\frac{1}{2} \sum_{l=1}^{\infty} \sum_{m+m^{\prime}=l-1}(-1)^{m+1} \sum_{\mu, \nu}\left(r_{l}\right)^{\mu \nu}\left\langle\tau_{m, \mu} \tau_{m^{\prime}, \nu} \tau_{d_{1}, \mu_{1}} \ldots \tau_{d_{n}, \mu_{n}}\right\rangle_{g-1} \\
& +\frac{1}{2} \sum_{l=1}^{\infty} \sum_{m+m^{\prime}=l-1}(-1)^{m+1} \sum_{\substack{g_{1}+g_{2}=g \\
I \sqcup J=\{1, \ldots, n\}}} \sum_{\mu, \nu}\left(r_{l}\right)^{\mu \nu}\left\langle\tau_{m, \mu} \prod_{i \in I} \tau_{d_{i}, \mu_{i}}\right\rangle_{g_{1}}\left\langle\tau_{m^{\prime}, \nu} \prod_{i \in J} \tau_{d_{i}, \mu_{i}}\right\rangle .
\end{aligned}
$$

REMARK 4.4. - One can easily deduce from these formulas that the action of the upper triangular group preserves the tameness property and the property of being an ancestor potential. 
Proposition 4.5 (based on [25], Section 6). - Let L be a linear combination of dual graphs and $r$ an element of the upper triangular Lie subalgebra of Givental's Lie algebra. Let $F$ be a formal tame ancestor potential. Then

$$
r .\left\langle p^{*}(L) \prod_{i=1}^{n+n^{\prime}} \psi_{i}^{d_{i}} \mathrm{ev}_{i}^{*}\left(\mu_{i}\right)\right\rangle_{g}
$$

is a linear combination of polynomials in correlators of the form

$$
\text { (i) }\left\langle p^{*}(L) \prod_{i=1}^{n+n^{\prime}+1} \psi_{i}^{d_{i}} \operatorname{ev}_{i}^{*}\left(\mu_{i}\right)\right\rangle_{g},
$$

where $d_{n+n^{\prime}+1} \geq 2$ and $\mu_{n+n^{\prime}+1}$ is a primary field;

$$
\text { (ii) }\left\langle p^{*}(L) \prod_{i=1}^{n+n^{\prime}} \psi_{i}^{d_{i}^{\prime}} \operatorname{ev}_{i}^{*}\left(\mu_{i}^{\prime}\right)\right\rangle_{g}
$$

where $d_{i}^{\prime}=d_{i}, \mu_{i}^{\prime}=\mu_{i}$ for all $i$ except one, and $d_{i}^{\prime}>d_{i}$ for one $i$;

$$
\text { (iii) }\left\langle p^{*}\left(\tau_{l}(L)\right) \prod_{i=1}^{n+n^{\prime}} \psi_{i}^{d_{i}} \operatorname{ev}_{i}^{*}\left(\mu_{i}\right) \cdot \operatorname{ev}_{\alpha}^{*}\left(\mu_{\alpha}\right) \operatorname{ev}_{\beta}^{*}\left(\mu_{\beta}\right)\right\rangle_{g},
$$

where $l \geq 1$ and $\mu_{\alpha}, \mu_{\beta}$ are primary fields;

$$
\text { (iv) }\left\langle\tau_{d_{\alpha}, \mu_{\alpha}} \prod_{i \in I} \tau_{d_{i}, \mu_{i}}\right\rangle_{0}\left\langle p^{*}(L) \psi_{\beta}^{d_{\beta}} \operatorname{ev}_{\beta}^{*}\left(\mu_{\beta}\right) \prod_{i \in J} \psi_{i}^{d_{i}} \operatorname{ev}_{i}^{*}\left(\mu_{i}\right)\right\rangle_{g},
$$

where $I \subset\left\{n+1, \ldots, n+n^{\prime}\right\}, I \sqcup J=\left\{1, \ldots, n+n^{\prime}\right\}, d_{\alpha}$ and $d_{\beta}$ are nonnegative integers, and $\mu_{\alpha}, \mu_{\beta}$ are primary fields;

$$
\text { (v) }\left\langle\tau_{d_{\alpha}, \mu_{\alpha}} \tau_{d_{j}, \mu_{j}} \prod_{i \in I} \tau_{d_{i}, \mu_{i}}\right\rangle_{0}\left\langle p^{*}(L) \psi_{\beta}^{d_{\beta}} \operatorname{ev}_{\beta}^{*}\left(\mu_{\beta}\right) \prod_{i \in J} \psi_{i}^{d_{i}} \operatorname{ev}_{i}^{*}\left(\mu_{i}\right)\right\rangle_{g},
$$

where $I \subset\left\{n+1, \ldots, n+n^{\prime}\right\}, j \in\{1, \ldots, n\}, I \sqcup J \sqcup\{j\}=\left\{1, \ldots, n+n^{\prime}\right\}, d_{\alpha}$ and $d_{\beta}$ are nonnegative integers, and $\mu_{\alpha}, \mu_{\beta}$ are primary fields.

REMARK 4.6. - If we work over a Novikov ring, we must introduce a summation over $D_{1}+D_{2}=D$ in the last term of the equality in Proposition 4.3 as well as in terms (iv) and (v) of Proposition 4.5.

COROLlary 4.7. - Let L be a linear combination of genus $g$ dual graphs with $n$ tails such that $\tau_{k}(L)$ is a universal relation for all $k$. Assume that $F_{L}^{\mathrm{pt}}$ vanishes. Then $F_{L}$ also vanishes for every $F$ that can be obtained from $F_{0}^{\alpha_{1}, \ldots, \alpha_{k}}$ by the upper triangular group action.

Proof. - The polynomials (i) to (v) are either entries of $F_{L}$ or of $F_{\tau_{l}(L)}$. In the latter case they vanish by assumption. Therefore the vector $F_{L}$ on the orbit $e^{t \widehat{r}} e^{F}$ is the solution of a linear differential equation in $t$ with vanishing initial conditions. Thus $F_{L}=0$ on the whole orbit. 
Proof of Proposition 4.5. - 1. We first prove the proposition in the particular case $n^{\prime}=0$, $d_{1}=\cdots=d_{n}=0$. In Section 2.1 .3 we gave a three steps algorithm to determine the linear combination of graphs $p^{*}(L) \prod_{i=1}^{n+n^{\prime}} \psi_{i}^{d_{i}} \mathrm{ev}_{i}^{*}\left(\mu_{i}\right)$. In our particular case, the steps are greatly simplified: Step 1 (replacing $\kappa$-classes by additional tails) remains unchanged; Step 2 (expressing ancestor $\psi$-classes in terms of descendant $\psi$-classes) can be skipped, since all genus zero 2-point correlators vanish for an ancestor potential and since there are no additional marked points; Step 3 (adding tails from $n+1$ to $n+n^{\prime}$ and multiplying by $\left.\psi_{i}^{d_{i}} \mathrm{ev}_{i}^{*}\left(\mu_{i}\right)\right)$ reduces to assigning the markings $\mu_{1}, \ldots, \mu_{n}$ to tails 1 to $n$.

As for the claim of our proposition, if $n^{\prime}=0$, then terms (iv) and (v) disappear, because $I \subset\left\{n+1, \ldots, n+n^{\prime}\right\}$ is then empty, and an ancestor potential does not have genus 0 correlators with fewer than 3 entries. Thus we must prove that $r .\left\langle p^{*}(L) \prod_{i=1}^{n} \operatorname{ev}_{i}^{*}\left(\mu_{i}\right)\right\rangle_{g}$ is a linear combination of terms (i), (ii), and (iii).

Let $G$ be a graph in $L$.

1a. First suppose that $G$ has only one vertex (of genus $g$ and valency $n$ ) with a class $\kappa_{k_{1}, \ldots, k_{m}}$ assigned to the vertex and classes $\psi_{i}^{d_{i}}$ assigned to the tails. Applying Steps 1 and 3 we obtain

$$
\left\langle p^{*}(G) \prod_{i=1}^{n} \mathrm{ev}_{i}^{*}\left(\mu_{i}\right)\right\rangle=\left\langle\prod_{i=1}^{m} \tau_{k_{i}+1,1} \prod_{i=1}^{n} \tau_{d_{i}, \mu_{i}}\right\rangle .
$$

To determine the action of $r$ on the correlator in the right-hand side we apply Equation (4) to it.

Applying the third and fourth terms in Equation (4) gives us term (iii) involving $\tau_{l}(G)$. Note that when we apply the fourth term of Equation (4), the indices $k_{1}, \ldots, k_{m}$ are distributed among the two correlators in all possible ways, according to the description of $\tau_{l}$.

Now apply the first two terms of Equation (4) to our correlator. We regroup them in the following way:

$$
\begin{gathered}
\sum_{l=1}^{\infty} \sum_{\mu}\left(r_{l}\right)_{\mu}^{1}\left[\sum_{j=1}^{m}\left\langle\tau_{k_{j}+l+1, \mu} \prod_{i \neq j} \tau_{k_{i}+1,1} \prod_{i=1}^{n} \tau_{d_{i}, \mu_{i}}\right\rangle-\left\langle\tau_{l+1, \mu} \prod_{i=1}^{m} \tau_{k_{i}+1,1} \prod_{i=1}^{n} \tau_{d_{i}, \mu_{i}}\right\rangle\right] \\
+\sum_{j=1}^{n}\left\langle r_{l}\left(\tau_{d_{j}, \mu_{j}}\right) \prod_{i=1}^{m} \tau_{k_{i}+1,1} \prod_{i \neq j} \tau_{d_{i}, \mu_{i}}\right\rangle .
\end{gathered}
$$

Consider the forgetful map $\pi: \bar{M}_{g, n+1} \rightarrow \bar{M}_{g, n}$. The pull-back $\pi^{*}\left(\kappa_{k_{1}, \ldots, k_{m}}\right)$ given by Lemma 2.1 imitates the expression in square brackets of (5). In addition, we have $\pi^{*}\left(\psi_{i}^{d_{i}}\right) \psi_{n+1}^{l+1}=\psi_{i}^{d_{i}} \psi_{n+1}^{l+1}$. It follows that the terms in square brackets add up to give term (i).

The last term of Equation (5) replaces one of the symbols $\tau_{d_{j}, \mu_{j}}$ by $\tau_{d_{j}^{\prime}, \mu_{j}^{\prime}}$ with $d_{j}^{\prime}>d_{j}$, which gives us term (ii).

1b. Now let $G$ be an arbitrary dual graph. Then the terms in square brackets of Equation (5) will appear for each vertex of the graph and their sum will still represent the class $\pi^{*}(G) \cdot \psi_{n+1}^{l+1} \mathrm{ev}_{n+1}^{*}(\mu)$.

The last term of Equation (5) replaces as before, one of the symbols $\tau_{d_{j}, \mu_{j}}$ by $\tau_{d_{j}^{\prime}, \mu_{j}^{\prime}}$. But now this symbol can either correspond to a tail or to a half-edge of $G$. If the symbol we replace corresponds to a tail, it gives rise to term (ii) in the proposition. If it corresponds to 
a half-edge of $G$, the corresponding term $\left\langle r_{l}\left(\tau_{d_{j}, \mu_{j}}\right) \prod_{i=1}^{m} \tau_{k_{i}+1,1} \prod_{i \neq j} \tau_{d_{i}, \mu_{i}}\right\rangle$ contributes to term (iii) of the proposition. Finally, the third and fourth terms in Equation (4) complete the expression for term (iii).

2. Now we return to the general case, when $n^{\prime}$ and $d_{1}, \ldots, d_{n+n^{\prime}}$ are arbitrary. Consider the forgetful maps

$$
\overline{\mathcal{M}}_{g, n+n^{\prime}+1} \stackrel{\pi_{1}}{\rightarrow} \overline{\mathcal{M}}_{g, n+n^{\prime}} \stackrel{\pi_{2}}{\rightarrow} \overline{\mathcal{M}}_{g, n}
$$

and the composition $\pi=\pi_{2} \circ \pi_{1}$.

If $L$ is our initial linear combination of dual graphs, then $L^{\prime}=\pi_{2}^{*}(L) \prod_{i=1}^{n+n^{\prime}} \psi_{i}^{d_{i}}$ is a welldefined linear combination of dual graphs obtained by the usual three steps algorithm, but without attaching the markings $\mu_{i}$ to the tails.

Now we can apply the particular case that we have just proved to the linear combination $L^{\prime}$. All we have to do is reinterpret the answer in terms of $L$. We claim the following.

Term (i) for $L^{\prime}$ gives term (i) for $L$. Indeed, the classes on the moduli spaces of curves involved in this term are

$$
\pi_{1}^{*}\left(L^{\prime}\right) \psi_{n+n^{\prime}+1}^{d_{n+n^{\prime}+1}}=\pi^{*}(L) \pi_{1}^{*}\left(\prod_{i=1}^{n+n^{\prime}} \psi_{i}^{d_{i}}\right) \psi_{n+n^{\prime}+1}^{d_{n+n^{\prime}+1}} .
$$

But since $d_{n+n^{\prime}+1} \geq 2$, we have $\pi_{1}^{*}\left(\psi_{i}^{d_{i}}\right) \psi_{n+n^{\prime}+1}^{d_{n+n^{\prime}+1}}=\psi_{i}^{d_{i}} \psi_{n+n^{\prime}+1}^{d_{n+n^{\prime}+1}}$ on $\overline{\mathcal{M}}_{g, n+n^{\prime}+1}$.

Term (ii) for $L^{\prime}$ gives term (ii) for $L$. This is obvious.

Term (iii) for $L^{\prime}$ gives terms (iii), (iv), and (v) for $L$. Indeed, the space $\mathscr{D}_{g, n+n^{\prime}}$ has two kinds of irreducible components: those that project to a component of $\mathscr{D}_{g, n}$ under $\pi_{2}$ and those that project onto $\bar{M}_{g, n}$ under $\pi_{2}$. In the first case we obtain term (iii) of the proposition. As to the second case, it occurs when the generic curve of the component of $\mathscr{D}_{g, n+n^{\prime}}$ has one component of genus $g$ and one component of genus 0 that is contracted by $\pi_{2}$. The class $\pi_{2}^{*}(L)$ is supported on the genus $g$ component. The contracted component of genus 0 contains either 0 or 1 point with markings $1, \ldots, n$ (those that are not forgotten by $\pi_{2}$ ). According to these two cases we obtain either term (iv) or term (v) from the proposition.

\subsection{The lower triangular group}

While the action of the upper triangular group modifies the Frobenius manifold, the action of the lower-triangular group preserves the Frobenius manifold, but incorporates 1and 2-point genus 0 correlators into the Gromov-Witten potential while simultaneously reexpanding it at a different point of the Frobenius manifold. More precisely, as we will see below, a translation of the variables $t_{0}^{\mu}$ is part of the action of the lower-triangular group.

The element $s=\sum_{l \geq 1} s_{l} z^{-l}$ acts on the series $e^{F}$ via the first order differential operator $\widehat{s}$ written out in Section 1.4.1.

In this expression we can omit the term $-\frac{1}{2 \hbar}\left(s_{3}\right)_{1,1}$. Indeed, it commutes with all other terms and the action of its exponential only adds the constant $\left(s_{3}\right)_{1,1}$ to $F_{0}$. Similarly, in the sequel we will consider $F_{0}$ to be defined up to an additive constant and omit those terms in differential operators that do nothing more than changing this constant. 
One more term of $\widehat{s}$ deserves special attention: the term

$$
\widehat{u}=\sum_{\mu}\left(s_{1}\right)_{1}^{\mu} \frac{\partial}{\partial t_{0}^{\mu}}
$$

is simply a partial derivative in the direction $s_{1}(1)$. The exponential $\exp (\widehat{u})$ is then a shift of the coordinates $t_{0}^{\mu}$ in this direction. A shift of coordinates is not a well-defined operation for formal power series. In our case, we consider power series in variables $t_{1}^{\mu}, t_{2}^{\mu}, \ldots$, whose coefficients are analytic in $t_{0}^{\mu}$ on the Frobenius manifold except perhaps its discriminant. Thus the shift is a well-defined operation, but it can, in some cases, lead us out of the realm of power series. In this case the genus expansion of the genus 0 Gromov-Witten potential will be a power series in $t_{1}^{\mu}, t_{2}^{\mu}, \ldots$, whose coefficients are analytic functions in $t_{0}^{\mu}$ with a singularity at the origin. (See, for instance, [5], Section 6, where $F$ and $G$ are the Gromov-Witten potentials without descendants for genus 0 and 1 respectively.)

We now need to give a proper definition of the action of $S=\exp (s)$. We have, up to omitted constant terms,

$$
\begin{aligned}
{[\widehat{s}, \widehat{u}] } & =\frac{1}{\hbar} \sum_{d, \mu}\left(s_{1} s_{d+1}\right)_{1, \mu} t_{d}^{\mu}, \\
{[\widehat{s},[\widehat{s}, \widehat{u}]] } & =\frac{1}{\hbar} \sum_{l, d, \mu}\left(s_{l} s_{1} s_{d+1}\right)_{1, \mu} t_{d+l}^{\mu}, \\
{[\widehat{s},[\widehat{s},[\widehat{s}, \widehat{u}]]] } & =\frac{1}{\hbar} \sum_{l_{1}, l_{2}, d, \mu}\left(s_{l_{1}} s_{l_{2}} s_{1} s_{d+1}\right)_{1, \mu} t_{d+l_{1}+l_{2}}^{\mu},
\end{aligned}
$$

etc. On the other hand, $[\widehat{u},[\widehat{u}, \widehat{s}]]=0$ (again, up to constant term). Thus, according to the Baker-Campbell-Hausdorff formula, we have $e^{\widehat{s}}=e^{-\widehat{u}} e^{\widehat{v}}$, where

$$
\begin{gathered}
\widehat{v}=\widehat{u}+\widehat{s}-\frac{1}{2}[\widehat{s}, \widehat{u}]+\frac{1}{12}[\widehat{s},[\widehat{s}, \widehat{u}]]-\frac{1}{720}[\widehat{s},[\widehat{s},[\widehat{s}, \widehat{u}]]]+\ldots \\
=\sum_{\substack{d, l \\
\mu, \nu}}\left(s_{l}\right)_{\nu}^{\mu} t_{d+l}^{\nu} \frac{\partial}{\partial t_{d}^{\mu}}+\frac{1}{2 \hbar} \sum_{\substack{d_{1}, d_{2} \\
\mu_{2}, \mu_{2}}}(-1)^{d_{1}}\left(s_{d_{1}+d_{2}+1}\right)_{\mu_{1}, \mu_{2}} t_{d_{1}}^{\mu_{1}} t_{d_{2}}^{\mu_{2}}+\sum C_{d, \mu} t_{d}^{\mu},
\end{gathered}
$$

where every $C_{d, \mu}$ is a finite polynomial in the matrix elements $\left(s_{l}\right)_{\mu \nu}$.

The following proposition describes the action of $\widehat{v}$ on individual correlators.

Proposition 4.8. - For $2-2 g-n<0$, we have

$$
\widehat{v} .\left\langle\tau_{d_{1}, \mu_{1}} \ldots \tau_{d_{n}, \mu_{n}}\right\rangle_{g}=\sum_{l=1}^{\infty} \sum_{i=1}^{n}\left\langle\tau_{d_{1}, \mu_{1}} \ldots s_{l}\left(\tau_{d_{i}, \mu_{i}}\right) \ldots \tau_{d_{n}, \mu_{n}}\right\rangle_{g} .
$$

If $g=0$ and $n=2$, we have

$$
\begin{aligned}
& \widehat{v} \cdot\left\langle\tau_{d_{1}, \mu_{1}} \tau_{d_{2}, \mu_{2}}\right\rangle_{0}= \\
& \sum_{l=1}^{\infty}\left[\left\langle s_{l}\left(\tau_{d_{1}, \mu_{1}}\right) \tau_{d_{2}, \mu_{2}}\right\rangle_{0}+\left\langle\tau_{d_{1}, \mu_{1}} s_{l}\left(\tau_{d_{2}, \mu_{2}}\right)\right\rangle_{0}\right]+(-1)^{d_{1}}\left(s_{d_{1}+d_{2}+1}\right)_{\mu_{1} \mu_{2}} .
\end{aligned}
$$


REMARK 4.9. - The action of $\widehat{v}$ on 1-point genus 0 correlators involves the coefficients $C_{d}^{\mu}$ and is much more complicated. However it is easy to see that such correlators never appear in the induced vectors of tautological relations. Therefore the action of $\widehat{v}$ on them is immaterial to us.

Lemma 4.10. - The action of $e^{\widehat{v}}$ is well-defined on power series; in other words, every coefficient in the series $e^{\widehat{v}} e^{F}$ is a finite polynomial in coefficients of $F$.

Proof. - The action of $\widehat{v}$ on a correlator is a polynomial involving correlators with strictly smaller $\sum d_{i}$ and matrix elements of the matrices $s_{l}$.

Definition 4.11. - The action of $e^{\widehat{s}}$ on $e^{F}$ is defined as the action of $e^{\widehat{v}}$ followed by a translation of coordinates $t_{0}^{\mu}$ by the vector $-\left(s_{0}\right)_{1}^{\mu}$.

To sum up: the same element $s$ of the lower triangular Lie subalgebra of Givental's Lie algebra determines three differential operators: $\widehat{s}, \widehat{u}$, and $\widehat{v}$. They have the following properties: the action of $e^{\widehat{v}}$ is well-defined on power series; the action of $e^{\widehat{u}}$ is a translation of coordinates; we have $e^{\widehat{s}}=e^{-\widehat{u}} e^{\widehat{v}}$.

Now let $L$ be a linear combination of dual graphs of genus $g$ with $n$ tails and $\left\langle p^{*}(L) \prod_{i=1}^{n+n^{\prime}} \psi_{i}^{d_{i}} \mathrm{ev}_{i}^{*}\left(\mu_{i}\right)\right\rangle_{g}$ an entry of its induced vector. Let $s$ be an element of the lower triangular Lie subalgebra of Givental's Lie algebra and $\widehat{v}$ the operator defined by Equation (6).

Proposition 4.12. - The result of the action

$$
\widehat{v} .\left\langle p^{*}(L) \prod_{i=1}^{n+n^{\prime}} \psi_{i}^{d_{i}} \mathrm{ev}_{i}^{*}\left(\mu_{i}\right)\right\rangle_{g}
$$

is a linear combination of polynomials in correlators of the form

$$
\left\langle p^{*}(L) \prod_{i=1}^{n+n^{\prime}} \psi_{i}^{d_{i}^{\prime}} \mathrm{ev}_{i}^{*}\left(\mu_{i}^{\prime}\right)\right\rangle_{g}
$$

with $d_{i}^{\prime}=d_{i}, \mu_{i}^{\prime}=\mu_{i}$ for all $i$ except one, while $d_{i}^{\prime}<d_{i}$ for one $i$.

COROLlary 4.13. - Suppose a formal Gromov-Witten potential $F$ is regular at the origin. If $F$ satisfies the equations of the family $\mathrm{PDE}_{L}$ for some tautological relation $L$ then the same is true of all formal Gromov-Witten potentials obtained from $F$ by the action of Givental's lower triangular group.

Proof. - Saying that $F$ is a solution of $\mathrm{PDE}_{L}$ is the same as saying that $F_{L}$ vanishes, because $F$ can, by assumption, be decomposed into a power series at the origin. The polynomials (9) are themselves entries of $F_{L}$. Therefore the vector $F_{L}$ on the orbit $e^{t \widehat{v}} e^{F}$ is the solution of a linear differential equation with vanishing initial conditions. Thus $F_{L}=0$ on the whole orbit.

On the other hand, since the differential equations in $\mathrm{PDE}_{L}$ have constant coefficients, these equations are preserved by translations of $t_{0}^{\mu}$. It follows that the condition $\operatorname{PDE}_{L}(F)=0$ is preserved by the action of the lower triangular group.

$4^{\mathrm{e}}$ SÉRIE - TOME $43-2010-\mathrm{N}^{\mathrm{o}} 4$ 
Proof of Proposition 4.12. - We must compute

$$
\widehat{v} \cdot\left\langle p^{*}(L) \prod_{i=1}^{n+n^{\prime}} \psi_{i}^{d_{i}} \operatorname{ev}_{i}^{*}\left(\mu_{i}\right)\right\rangle_{g} .
$$

The main element of the proof is the following observation: every contribution of the second term in Equation (8) cancels with some contribution of the first term in Equations (7) and (8).

Indeed, recall that in the definition of the induced vector $F_{L}$ every half-edge and every tail of each graph in $L$ was replaced by a "stick" of several edges (Step 2), and then new tails were added in all possible ways to the new graphs (Step 3). Consider the linear combination $L_{3}$ of graphs obtained after Step 3 of the procedure. In $L_{3}$ take two graphs $\Gamma_{1}$ and $\Gamma_{2}$ differing only in one fragment:

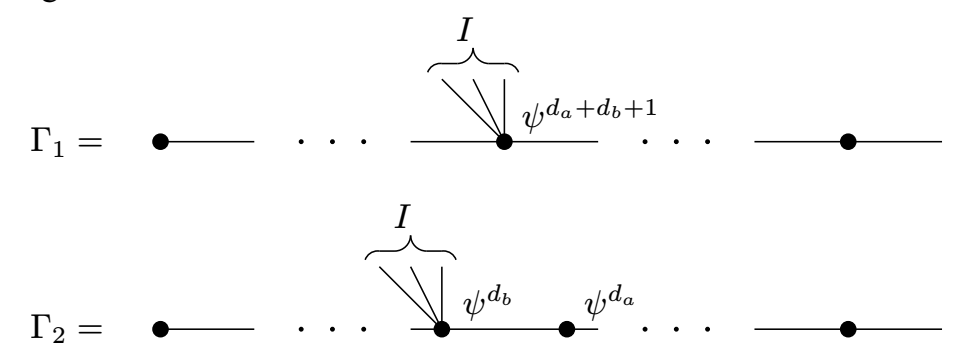

Here $I \subset\left\{n+1, \ldots, n+n^{\prime}\right\}$ is a set of labels of the tails added in Step 3 .

The polynomial $P_{\Gamma_{1}}$ contains the factor

$$
Q_{\Gamma_{1}}=\left\langle\tau_{d_{a}+d_{b}+1, \mu_{b}} \tau_{0, \nu_{b}} \prod_{i \in I} \tau_{d_{i}, \mu_{i}}\right\rangle_{0}
$$

The polynomial $P_{\Gamma_{2}}$ contains the factor

$$
Q_{\Gamma_{2}}=\sum_{\mu_{a}, \nu_{a}}\left\langle\tau_{d_{a}, \mu_{a}} \tau_{0, \nu_{a}}\right\rangle_{0} \eta^{\nu_{a} \mu_{b}}\left\langle\tau_{d_{b}, \mu_{b}} \tau_{0, \nu_{b}} \prod_{i \in I} \tau_{d_{i}, \mu_{i}}\right\rangle_{0}
$$

Apply the operator $\widehat{v}$ to the correlator $\left\langle\tau_{d_{a}, \mu_{a}} \tau_{0, \nu_{a}}\right\rangle_{0}$ in the expression $Q_{\Gamma_{2}}$, and take the contribution of the second term in Equation (8). We obtain

$$
\begin{aligned}
(-1)^{d_{a}} \sum_{\mu_{a}, \nu_{a}}\left(s_{d_{a}+1}\right)_{\mu_{a} \nu_{a}} \eta^{\nu_{a} \mu_{b}} & \left\langle\tau_{d_{b}, \mu_{b}} \tau_{0, \nu_{b}} \prod_{i \in I} \tau_{d_{i}, \mu_{i}}\right\rangle_{0} \\
& =\left\langle s_{d_{a}+1}\left(\tau_{d_{a}+d_{b}+1, \mu_{b}}\right) \tau_{0, \nu_{b}} \prod_{i \in I} \tau_{d_{i}, \mu_{i}}\right\rangle_{0}
\end{aligned}
$$

But this term is part of the action of $\widehat{v}$ on $P_{\Gamma_{1}}$. Indeed, it is part of the sum in the first term of Equations (7) and (8) applied to

$$
\left\langle\tau_{d_{a}+d_{b}+1, \mu_{b}} \tau_{0, \nu_{b}} \prod_{i \in I} \tau_{d_{i}, \mu_{i}}\right\rangle_{0}
$$

Since $\Gamma_{2}$ has one edge more than $\Gamma_{1}$, these terms appear with opposite signs in Substitution (1) and hence cancel.

Let us look at the contributions of the first term of Equations (7) and (8) that survived the cancellation. They are exactly those where $s_{l}$ is applied to the symbols $\tau_{d, \mu}$ corresponding to the $n+n^{\prime}$ marked points and such that $l$ is smaller than or equal to the corresponding $d_{i}$. These contributions combine into the expression given in the proposition. 
Proof of Theorem 4. - The result follows from Corollaries 4.7 and 4.13.

REMARK 4.14. - Unfortunately, for the time being we cannot prove the implication ( $L$ is a universal relation) $\Rightarrow\left(\tau_{1}(L)\right.$ is a universal relation). There are two reasons for that. First, as explained in Remark 2.7, for not necessarily connected graphs checking that $F_{\tau_{1}(L)}=0$ does not imply that $\tau_{1}(L)$ is a universal relation. Second, term (iii) of Proposition 4.5 does not allow us to multiply $\tau_{1}(L)$ by arbitrary powers of $\psi_{\alpha}$ and $\psi_{\beta}$, but only by some of their combinations, namely, the classes $\rho_{k}$ of Section 2.3.1.

\section{Acknowledgements}

The authors are deeply grateful to M. Kazarian for his explanations on Frobenius manifolds and Givental's quantization. We thank B. Dubrovin for pointing out the necessity of analyticity conditions on Gromov-Witten potentials. The third author benefited greatly from a discussion with Tom Coates. We also wish to thank the participants of the Moduli Spaces program at the Mittag-Leffler Institute (Djursholm, Sweden) for the stimulating atmosphere and lots of interesting discussions. We thank the Institute for hospitality and support.

C. Faber is supported by the grants 622-2003-1123 from the Swedish Research Council and DMS-0600803 from the National Science Foundation and by the Göran Gustafsson Foundation for Research in Natural Sciences and Medicine. S. Shadrin is supported by the Vidi grant of the Netherlands Organization for Scientific Research. D. Zvonkine is partly supported by the ANR project "Geometry and Integrability in Mathematical Physics" ANR-05-BLAN-0029-01.

\section{REFERENCES}

[1] D. Abramovich, T. J. Jarvis, Moduli of twisted spin curves, Proc. Amer. Math. Soc. 131 (2003), 685-699 (electronic).

[2] L. Caporaso, C. Casagrande, M. Cornalba, Moduli of roots of line bundles on curves, Trans. Amer. Math. Soc. 359 (2007), 3733-3768 (electronic).

[3] A. Chiodo, The Witten top Chern class via K-theory, J. Algebraic Geom. 15 (2006), 681-707.

[4] A. Chiodo, Stable twisted curves and their $r$-spin structures, preprint arXiv:math.AG/0603687.

[5] B. Dubrovin, Y. Zhang, Bi-Hamiltonian hierarchies in 2D topological field theory at one-loop approximation, Comm. Math. Phys. 198 (1998), 311-361.

[6] C. Faber, R. Pandharipande, Logarithmic series and Hodge integrals in the tautological ring, Michigan Math. J. 48 (2000), 215-252.

[7] C. Faber, R. Pandharipande, Relative maps and tautological classes, J. Eur. Math. Soc. (JEMS) 7 (2005), 13-49.

[8] A. B. Givental, Semisimple Frobenius structures at higher genus, Int. Math. Res. Not. 2001 (2001), 1265-1286.

$4^{\mathrm{e}}$ SÉRIE - TOME $43-2010-\mathrm{N}^{\mathrm{o}} 4$ 
[9] A. B. Givental, Symplectic geometry of Frobenius structures, Mosc. Math. J. 1 (2001), 551-568.

[10] A. B. Givental, $A_{n-1}$ singularities and $n \mathrm{KdV}$ hierarchies, Mosc. Math. J. 3 (2003), 475-505, 743.

[11] A. B. Givental, Gromov-Witten invariants and quantization of quadratic hamiltonians, in Frobenius manifolds, Aspects Math. E36, Vieweg, 2004, 91-112.

[12] T. Graber, R. PAndharipande, Constructions of nontautological classes on moduli spaces of curves, Michigan Math. J. 51 (2003), 93-109.

[13] T. Graber, R. Vakil, On the tautological ring of $\overline{\mathscr{M}}_{g, n}$, Turkish J. Math. 25 (2001), 237-243.

[14] R. Hain, E. Looijenga, Mapping class groups and moduli spaces of curves, in Algebraic geometry - Santa Cruz 1995, Proc. Sympos. Pure Math. 62, Amer. Math. Soc., 1997, 97-142.

[15] E.-N. Ionel, Topological recursive relations in $H^{2 g}\left(\mathscr{M}_{g, n}\right)$, Invent. Math. 148 (2002), $627-658$.

[16] T. J. JARVIS, Geometry of the moduli of higher spin curves, Internat. J. Math. 11 (2000), 637-663.

[17] T. J. Jarvis, T. Kimura, A. Vaintrob, Tensor products of Frobenius manifolds and moduli spaces of higher spin curves, in Conférence Moshé Flato 1999, Vol. II (Dijon), Math. Phys. Stud. 22, Kluwer Acad. Publ., 2000, 145-166.

[18] T. J. Jarvis, T. Kimura, A. Vaintrob, Gravitational descendants and the moduli space of higher spin curves, in Advances in algebraic geometry motivated by physics (Lowell, MA, 2000), Contemp. Math. 276, Amer. Math. Soc., 2001, 167-177.

[19] T. J. Jarvis, T. Kimura, A. Vaintrob, Moduli spaces of higher spin curves and integrable hierarchies, Compositio Math. 126 (2001), 157-212.

[20] T. J. Jarvis, T. Kimura, A. Vaintrob, Spin Gromov-Witten invariants, Comm. Math. Phys. 259 (2005), 511-543.

[21] M. Kontsevich, Intersection theory on the moduli space of curves and the matrix Airy function, Comm. Math. Phys. 147 (1992), 1-23.

[22] M. Kontsevich, Y. Manin, Gromov-Witten classes, quantum cohomology, and enumerative geometry, Comm. Math. Phys. 164 (1994), 525-562.

[23] Y.-P. LeE, Witten's conjecture and the Virasoro conjecture for genus up to two, in Gromov-Witten theory of spin curves and orbifolds, Contemp. Math. 403, Amer. Math. Soc., 2006, 31-42.

[24] Y.-P. LeE, Invariance of tautological equations. I. Conjectures and applications, J. Eur. Math. Soc. (JEMS) 10 (2008), 399-413.

[25] Y.-P. LeE, Invariance of tautological equations. II. Gromov-Witten theory, J. Amer. Math. Soc. 22 (2009), 331-352.

[26] Y.-P. LeE, Witten's conjecture, Virasoro conjecture, and invariance of tautological equations, preprint arXiv:math.AG/0311100.

[27] Y.-P. Lee, R. Pandharipande, Frobenius manifolds, Gromov-Witten theory, and Virasoro constraints, preprint http://www . math. princeton. edu/ ${ }^{\sim}$ rahulp/Part1. ps and http://www. math.princeton. edu/ rahulp/Part2.ps. 
[28] T. Mochizuki, The virtual class of the moduli stack of stable $r$-spin curves, Comm. Math. Phys. 264 (2006), 1-40.

[29] D. Mumford, Towards an enumerative geometry of the moduli space of curves, in Arithmetic and geometry, Vol. II, Progr. Math. 36, Birkhäuser, 1983, 271-328.

[30] A. Polishchuk, Witten's top Chern class on the moduli space of higher spin curves, in Frobenius manifolds, Aspects Math., E36, Vieweg, 2004, 253-264.

[31] A. Polishchuk, A. Vaintrob, Algebraic construction of Witten's top Chern class, in Advances in algebraic geometry motivated by physics (Lowell, MA, 2000), Contemp. Math. 276, Amer. Math. Soc., 2001, 229-249.

[32] S. ShADRIN, Geometry of meromorphic functions and intersections on moduli spaces of curves, Int. Math. Res. Not. 2003 (2003), 2051-2094.

[33] S. Shadrin, Intersections in genus 3 and the Boussinesq hierarchy, Lett. Math. Phys. 65 (2003), 125-131.

[34] S. Shadrin, D. Zvonkine, Intersection numbers with Witten's top Chern class, Geom. Topol. 12 (2008), 713-745.

[35] E. Witten, Algebraic geometry associated with matrix models of two-dimensional gravity, in Topological methods in modern mathematics (Stony Brook, NY, 1991), Publish or Perish, 1993, 235-269.

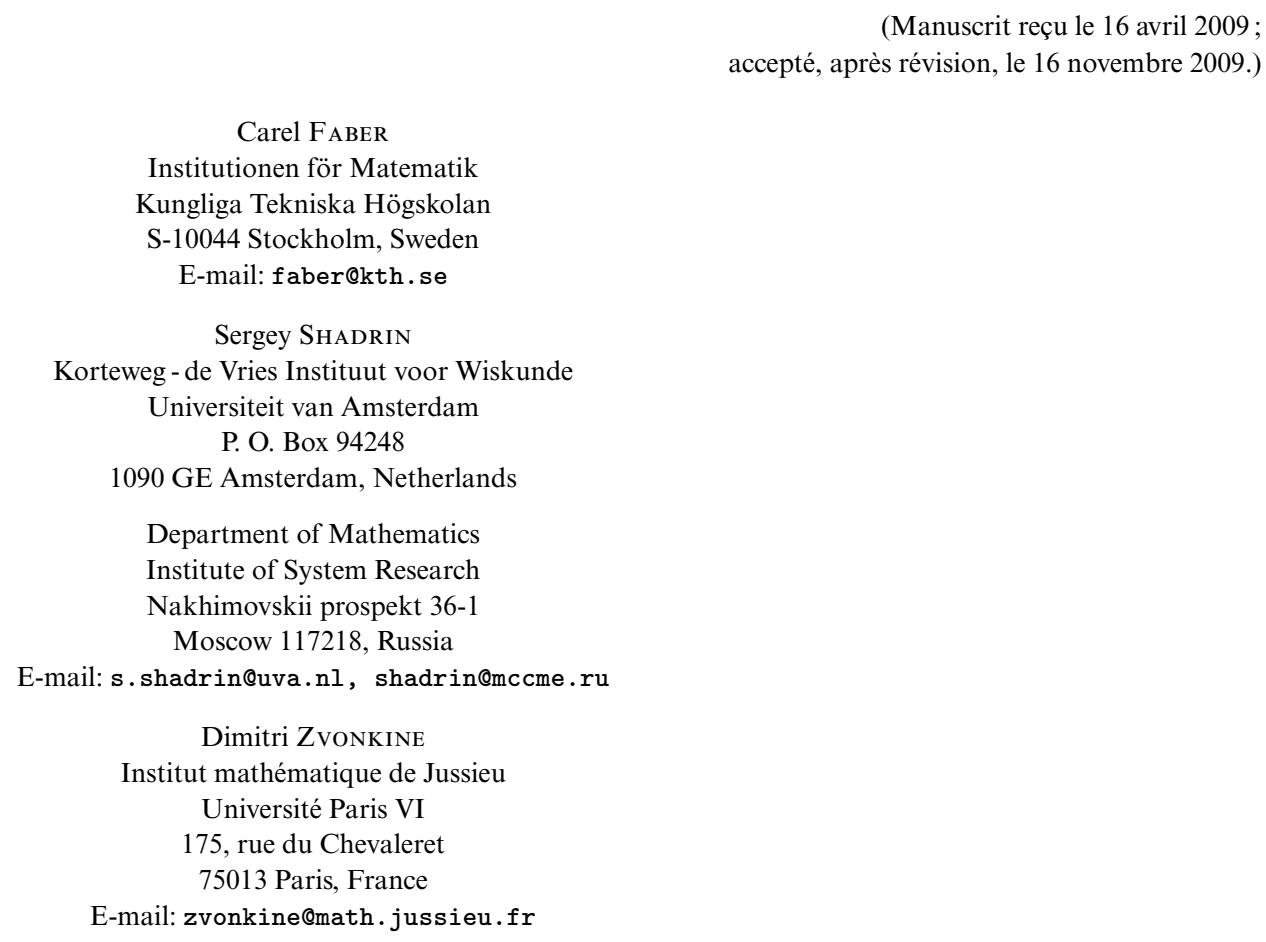

